\title{
Tagging Quantitative Trait Loci for Maturity-Corrected Late Blight Resistance in Tetraploid Potato with PCR-Based Candidate Gene Markers
}

\author{
Christina Angelika Bormann, ${ }^{1}$ Andreas Marcus Rickert, ${ }^{1}$ Rosa Angela Castillo Ruiz, ${ }^{1}$ Jürgen Paal, ${ }^{1}$ \\ Jens Lübeck, ${ }^{2}$ Josef Strahwald, ${ }^{2}$ Karsten Buhr, ${ }^{2}$ and Christiane Gebhardt ${ }^{1}$ \\ ${ }^{1}$ Max-Planck-Institute for Plant Breeding Research, Carl-von-Linné Weg 10, D-50829 Cologne, Germany; ${ }^{2}$ Saka-Ragis \\ Pflanzenzucht GbR, Zuchtstation Windeby, D-24340 Windeby bei Eckernförde, Germany
}

Submitted 12 November 2003. Accepted 16 May 2004.

Late blight caused by the oomycete Phytophthora infestans is the economically most important and destructive disease in potato cultivation. Quantitative resistance to late blight available in tetraploid cultivars is correlated with late maturity in temperate climates, which is an undesirable characteristic. A total of 30 DNA-based markers known to be linked to loci for pathogen resistance in diploid potato were selected and tested as polymerase chain reactionbased markers for linkage with quantitative trait loci (QTL) for late blight resistance and plant maturity in two half-sib families of tetraploid potatoes. Most markers originated from within or were physically closely linked to candidate genes for quantitative resistance factors. The families were repeatedly evaluated in the field for quantitative resistance to late blight and maturity. Resistance was corrected for the maturity effect. Nine of eleven different map segments tagged by the markers harbored QTL affecting maturity-corrected resistance. Interactions were found between unlinked resistance QTL, providing testable strategies for marker-assisted selection in tetraploid potato. Based on the linkage observed between QTL for resistance and plant maturity and based on the genetic interactions observed between candidate genes tagging resistance QTL, we discuss models for the molecular basis of quantitative resistance and maturity.

Additional keywords: late blight, marker-assisted selection (MAS).

The most important disease in potato cultivation worldwide is late blight, caused by the oomycete Phytophthora infestans. If not controlled, late blight epidemics can lead to complete yield loss. The first outbreak of late blight in the 19th century lead to one of the most destructive famines in Europe (Salaman 1985). Since then, resistance to late blight has been an important character in variety development. At the beginning of the 20th century, single genes for race-specific resistance to late blight ( $R$ genes) were discovered in the wild hexaploid potato species Solanum demissum. The introgression of $R$ genes from $S$. demissum into tetraploid potato cultivars resulted in nondurable resistance to late blight, because new races of $P$. infestans soon overcame this monogenic resistance (Ross 1986). Quantitative resistance to late blight is

Corresponding author: C. Gebhardt; E-mail: gebhardt@mpiz-koeln.mpg.de; Telephone: ++49 (0)221-5062-430; Fax: ++49 (0)221-5062-413. mostly race-nonspecific, controlled by more than one gene and, therefore, more durable (Wastie 1991). Genotypes with high levels of field resistance to late blight often have other, undesirable agronomic characteristics. In particular, such cultivars usually are late maturing under the long-day conditions of middle and northern Europe (Wastie 1991). The well-known correlation between field resistance to late blight and late maturity can have two reasons. Either both characters are pleiotropic effects of the same gene or genes or the genes that control resistance and plant maturity are different but closely linked (Colon et al. 1995). To break the correlation between resistance and late maturity, resistance factors that do not affect maturity or are separated by recombination from the genes controlling maturity must be identified.

QTL (quantitative trait loci) for late blight resistance have been mapped in a number of experimental populations of diploid potatoes, using DNA-based markers (Collins et al. 1999; Ewing et al. 2000; Ghislain et al. 2001; Leonards-Schippers et al. 1994; Oberhagemann et al. 1999; Sandbrink et al. 2000; Trognitz et al. 2002; Visker et al. 2003) and in one tetraploid population (Meyer et al. 1998). In these experiments, QTL for late blight resistance were identified on most of the 12 potato chromosomes (equal to one complement). Based on common restriction fragment length polymorphism (RFLP) markers that anchor together the homologous linkage groups of different mapping populations, some QTL were located in the same map segments in different genetic backgrounds (Oberhagemann et al. 1999; Simko 2002). The most persistent and most prominent QTL for late blight resistance is located on chromosome $\mathrm{V}$ in a map segment tagged by RFLP markers GP21 and GP179 (Leonards-Schippers et al. 1994; Oberhagemann et al. 1999; Simko 2002). Linked to the same RFLP markers is a major QTL for plant maturity that is not separable from the QTL for late blight resistance by linkage analysis (Collins et al. 1999; Oberhagemann et al. 1999; Visker et al. 2003). Effects on plant maturity were also linked to some but not all QTL for late blight resistance detected on other chromosomes. QTL alleles that increase foliage resistance to late blight delay plant maturity and vice versa, thereby explaining the observed phenotypic correlation between resistance to late blight and late maturity (Collins et al. 1999; Oberhagemann et al. 1999; Visker et al. 2003).

The molecular basis of the QTL for late blight resistance and maturity is not known. It was proposed, based on the observation of close linkage between a major QTL for late blight resistance and the $R l$ gene for race specific resistance to late blight on chromosome V, that the QTL for late blight resistance and $R l$ are related at the molecular level (Leonards- 
Schippers et al. 1992, 1994). Recently, the $R l$ gene was shown to be a member of the large family of plant resistance genes that have in common a putative nucleotide-binding (NB) domain and a leucine-rich-repeat (LRR) domain (Ballvora et al. 2002). Additional cases of colocalization of resistance QTL, $R$ loci, and genes with sequence similarity to cloned plant $R$ genes for pathogen resistance (resistance-gene-like [RGL] genes) were observed in the potato genome (Gebhardt and Valkonen 2001). Linkage between QTL for late blight resistance and pathogenesis-related (PR) genes has also been observed (Leonards-Schippers et al. 1994; Trognitz et al. 2002). Genes known or suspected to have a functional role in the expression of a phenotypic trait that colocalize with QTL for the same trait are considered as candidates for controlling the QTL (Pflieger et al. 2001). RGL and PR genes are therefore, besides unknown genes, known candidates for controlling quantitative resistance to late blight.

Here, we describe the tagging of QTL for late blight resistance and plant maturity in two tetraploid $S$. tuberosum breeding populations, based on genotyping with polymerase chain reaction (PCR)-based markers that were selected using the following criterion. The markers map to segments of the potato molecular genetic maps that have previously been shown in diploid mapping populations to contain factors controlling quantitative or qualitative resistance, or both, to pathogens.
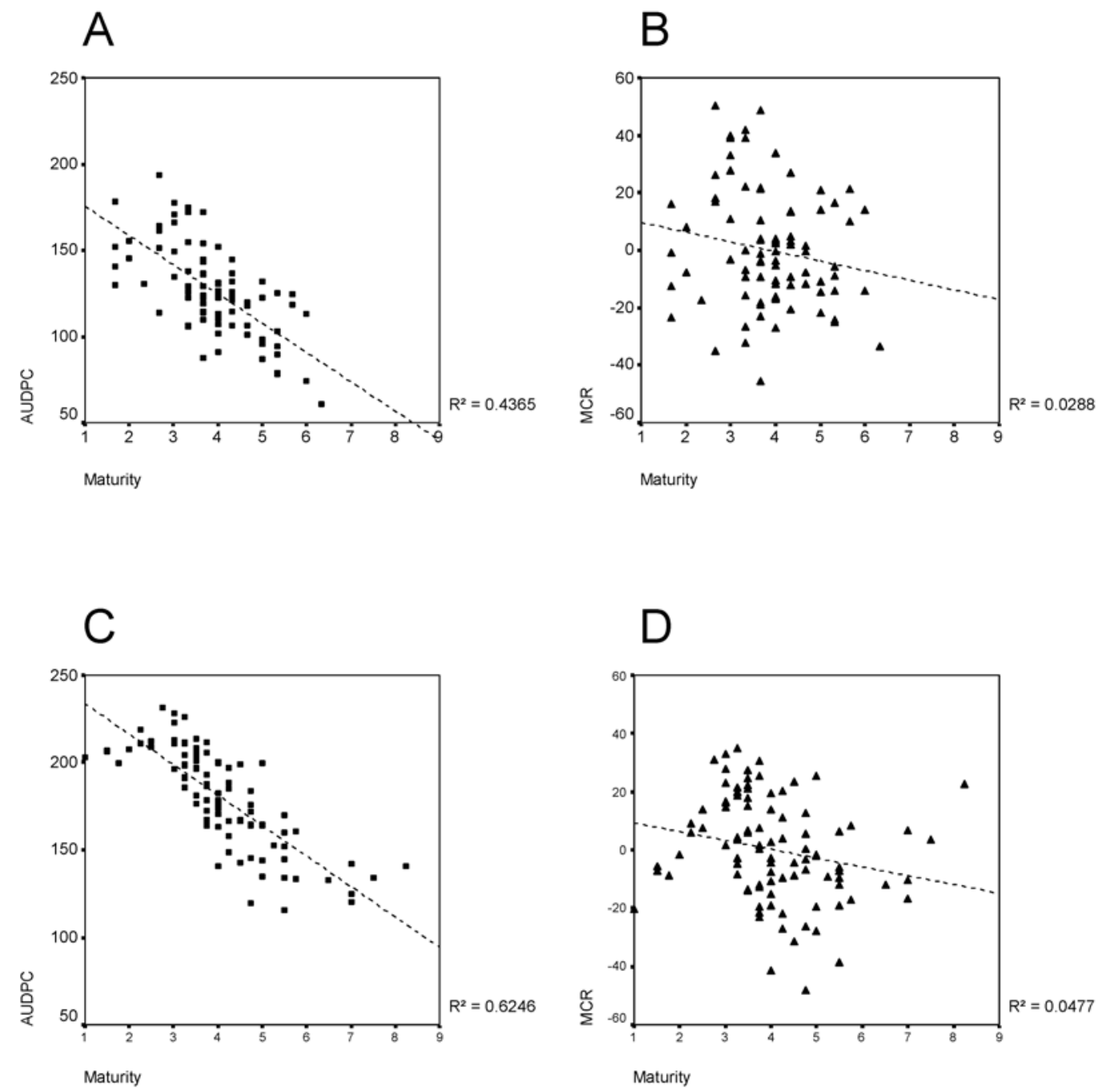

Fig. 1. Regression curves of maturity (mean values over all test years) against $\mathbf{A}$ and $\mathbf{C}$, area under disease progress curve (AUDPC) and $\mathbf{B}$ and $\mathbf{D}$, maturitycorrected resistance (MCR) (mean values over all test years). Data of the Escort population are shown in A and B, and that of the Nikita population are shown in $\mathrm{C}$ and $\mathrm{D}$.

Some of the markers originated from within or were physically tightly linked to RGL or PR candidate genes. Quantitative late blight resistance was evaluated in the field and was corrected for the influence of plant maturity, in order to identify QTL for late blight resistance having no effect on plant maturity. Models are discussed for the molecular basis of resistance QTL and for marker-assisted selection (MAS) of genotypes with increased resistance to late blight that is not compromised by late maturity.

\section{RESULTS}

Field evaluation

of late blight resistance and plant maturity.

Based on field evaluation of plant maturity in 1999, the plants of the Escort and Nikita family were ordered into an early maturing group (maturity scores from 1 to 3 ) and a late maturing group (maturity scores from 4 to 8 ). To ensure similar infection pressure on plants at a similar developmental stage, infection of the late maturing group was started one to two weeks later than infection of the early maturing group. Both families were evaluated in the field for late blight resistance in years 2000 and 2001. The Nikita family was evaluated a third time in 2002. In 2002, the infection level of $P$. infestans was higher than in the previous years. AUDPC (area under dis-

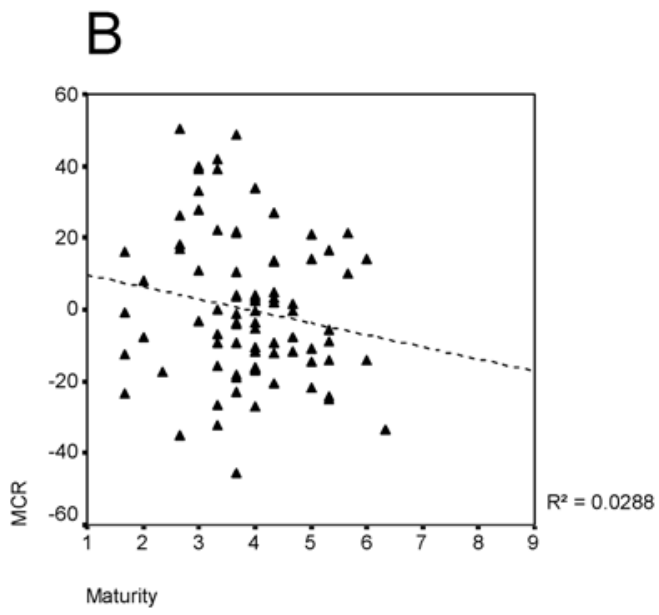


ease progress curve) was strongly correlated $(P \leq 0.01)$ with maturity in both families, with higher resistance (lower AUDPC values) being correlated with later maturity (Fig. 1A and C). Based on the means over all test years, the Escort family (Fig. 1A) was, on average, more resistant to late blight than the Nikita family (Fig. 1C). This was due to the higher infection level observed in the Nikita family in year 2002. The Escort family showed less variability for maturity (scores between 1.5 and 6.5) when compared with the Nikita family (scores between 1 and 8). Very late maturing genotypes (score 9) were absent from both families due to preselection applied against very late genotypes. Maturity-corrected resistance (MCR) was no longer correlated $(P>0.200)$ with maturity (Fig. 1B and D).

Analysis of variance of the average AUDPC and MCR data over two (Escort family) and three (Nikita family) years of testing resulted in significant genotype-by-genotype and genotype-by-year variation $(P \leq 0.01)$, whereas error variance was not significant. The mean-based heritability $\left(h^{2}\right)$ of AUDPC was 0.86 and 0.90 for the Escort and Nikita families, respectively. The heritability of MCR was lower, with $h^{2}=0.76$ for the Escort family and $h^{2}=0.87$ for the Nikita family.

\section{Genotypic analysis.}

PCR with 30 specific primer pairs (Table 1) resulted in clear amplicons in the two families. A total of 22 amplicons (73\%) were polymorphic in members of the Escort and Nikita families, either directly (six sequence characterized amplified region [SCAR] or allele-specific oligonucleotide [ASO] markers) or after restriction enzyme digestion and single strand conforma- tion polymorphism (SSCP) analysis (16 cleaved amplified polymorphic sequence [CAPS] or SSCP markers; Table 1). Three polymorphic SSCP markers (GP180, BA228D23t3, and BA1M23t3) were not scorable in the genotypes analyzed, due to insufficient fragment separation. The remaining 19 informative PCR-based markers $(63 \%)$ generated 60 segregating fragments, some of which were redundant or highly correlated with each other $(>90 \%)$, indicating close linkage in coupling phase between them. Counting such highly correlated fragments as one allele reduced the number of different marker alleles to fifty. The 50 marker alleles tagged 11 different map segments, one each on potato chromosomes II to IX and on chromosome XII and two on chromosome XI (Fig. 2).

Segregation of the marker alleles was tested for goodness of fit to one of four genetic models using the $\chi^{2}$ test. When the marker allele was present in both parents, the observed segregation ratio was tested for goodness of fit to the ratios $3: 1$ (simplex by simplex, presence vs. absence) or 11:1 (duplex by simplex). When the marker allele was present in only one parent, the models 1:1 (simplex by nulliplex) and 5:1 (duplex by nulliplex, presence vs. absence) were tested. Table 2 shows the results for the 40 marker alleles that had significant trait effects (Tables 3 to 7). Of the marker alleles, $70 \%$ fit one of the four genetic models tested. The segregation ratios of the remaining $30 \%$ were distorted.

\section{PCR-based markers with effects}

on late blight resistance and maturity.

When testing each marker allele individually, 21 of 50 marker alleles (43\%) tagging five and seven different map seg-

Table 1. Markers genotyped by polymerase chain reaction $(\mathrm{PCR})$ in populations Escort $\times$ Leyla and Nikita $\times$ Leyla

\begin{tabular}{|c|c|c|c|c|c|}
\hline $\mathbf{L G}$ & Marker & Primer sequence $5^{\prime} \rightarrow 3^{\prime}$, reference, accession number & $\mathbf{T}_{\mathbf{a}}\left({ }^{\circ} \mathbf{C}\right)$ & $\begin{array}{l}\text { PCR product } \\
\text { size (bp) }\end{array}$ & $\begin{array}{l}\text { Type of } \\
\text { polymorphism }\end{array}$ \\
\hline I & CP108 & ttt agc tgc ata caa gat gc (f), ccc aag gcc tag gcc c (r) & 52 & 450 & $-^{\mathrm{y}}$ \\
\hline II & GP23 & Oberhagemann et al. 1999 & 52 & $600 / 1200$ & $\mathrm{SSCP} / M s e \mathrm{I}$ \\
\hline III & GP25 & Oberhagemann et al. 1999 & 52 & 600 & $\begin{array}{l}\text { CAPS/TaqI, } \\
\text { SSCP/TaqI }\end{array}$ \\
\hline \multirow[t]{3}{*}{ IV } & P450 (-SSCP2) & $\begin{array}{l}\text { cat caa caa caa ctg tat ggg cta tgg (f), aaa gat cgt cet tac tag ccg cag (r), } \\
\text { accession no. U48434 }\end{array}$ & 56 & 560 & $\mathrm{SSCP} / A l u \mathrm{I}$ \\
\hline & GP180 & Oberhagemann et al. 1999 & 55 & 400 & $\mathrm{SSCP} / \mathrm{Mse}^{\mathrm{z}}$ \\
\hline & STM3016 & Milbourne et al. 1998 & 53 & $110 / 150$ & SCAR \\
\hline \multirow[t]{6}{*}{$\mathrm{V}$} & $\operatorname{Cos} \mathrm{A}$ & Gebhardt et al. 2003 & 55 & $210 / 250$ & SCAR \\
\hline & $\mathrm{R} 1$ & Ballvora et al. 2002 & 55 & 1400 & ASO \\
\hline & GP179 & Meksem et al. 1995 & 55 & $500 / 570$ & SCAR \\
\hline & BA121o1t7 & cat aaa gtc ttg cag agt tct caa (f), gga ttt gga gca ccg ggt tgt (r) & 55 & 233 & SSCP \\
\hline & P450 (-SSCP1) & Same as P450(-SSCP2) on LG IV & & & $\mathrm{SSCP} / A l u \mathrm{I}$ \\
\hline & CP113 & Niewöhner et al. 1995 & 45 & 900 & - \\
\hline \multirow[t]{2}{*}{ VI } & BA $1 \mathrm{~m} 23 \mathrm{t} 3$ & acc ctc cta ttt agt att gtt cag (f), gga aaa gat ata tag tgg ggt aca (r) & 55 & 270 & $\mathrm{SSCP}^{\mathrm{z}}$ \\
\hline & GP76 & Oberhagemann et al. 1999 & 52 & 800 & CAPS/RsaI \\
\hline \multirow[t]{2}{*}{ VII } & gro1-2-P40 & agt ata aca ggg ata tat gct c (f), tag gea tcc att tgt cga aaa g (r) & 58 & 500 & ASO \\
\hline & gro1-11-P41 & acc tga cta atg aag aag aag c (f), tca aag cag tag cac cca aac (r) & 58 & 600 & - \\
\hline \multirow[t]{5}{*}{ VIII } & RC5t7 & aga cca atg atg gag atg tat acg (f), cac acc tgg taa gga ttt aca tca (r) & 56 & 650 & - \\
\hline & $\mathrm{RC} 5 \mathrm{t} 3$ & gac cgt gtg cac gcg gag gag (f), tga att cgc ata tta atg gtt agc (r) & 56 & 900 & CAPS/RsaI \\
\hline & $\mathrm{RC} 1 \mathrm{t} 3$ & get tca cca tga aac ggg tct aca $t(f)$, cet ttc gac tta cat gac cat tac g (r) & 58 & 800 & - \\
\hline & 573 & cac agc ttg ata ttg tgg ttc tgc a (f), tga atc ctg agc aag caa gtt cag (r) & 56 & 500 & - \\
\hline & $57 \mathrm{t} 3$ & caa tcc gtt gga gtt tag acg atc (f), gaa gag gag aac ctt ggt gga tgg (r) & 58 & 800 & $\mathrm{SSCP} / A l u \mathrm{I}$ \\
\hline IX & Prp1 & Oberhagemann et al. 1999 , Taylor et al. 1990 & 55 & $200 / 600$ & SCAR \\
\hline \multirow[t]{5}{*}{$\mathrm{XI}$} & BA228d $23 \mathrm{t} 3$ & tcg tag gta aat ttt caa ggt cc (f), ctc ttc tcg gaa tga ttt aca ag (r) & 54 & 90 & $\mathrm{SSCP}^{\mathrm{z}}$ \\
\hline & GP125 & Oberhagemann et al. 1999 & 57 & 1000 & $\mathrm{SSCP} / M s e \mathrm{I}$ \\
\hline & NL25 & tat tgt taa tcg tta ctc cet c (f), aga gtc gtt tta ccg act cc (r) & 60 & $200 / 1000$ & $\mathrm{SSCP} / M s e \mathrm{I}$ \\
\hline & NL27 & Marczewski et al. 2001 & 62 & 1200 & $\mathrm{SSCP} / M s e \mathrm{I}$ \\
\hline & 683 & tga tgg tgc tgg tag agg ttc ttg (f), aag acc gat tgc ctg aag cat tag (r) & 58 & 600 & $\mathrm{SSCP} / A l u \mathrm{I}$ \\
\hline \multirow[t]{4}{*}{ XII } & E22 & gat ggt agt ggc cga ggc aaa tg (f), tcc agc agg aca tgt aaa caa act (r) & 56 & $400 / 600$ & - \\
\hline & 319 & gga cac gaa cct aca att gca gat g (f), ggt aat gtc aaa agt ggc agc atg agt (r) & 56 & 400 & - \\
\hline & GP34 & Bendahmane et al. 1997 & 52 & 850 & $\mathrm{SSCP} / M s e \mathrm{I}$ \\
\hline & $\mathrm{Rx} 1$ & ggg aga att ctc tgt ggc atg g (f), tat agc aag aat gag cat gca gg (r) & 56 & 771 & $\mathrm{SSCP} / \mathrm{Mse} \mathrm{I}$ \\
\hline
\end{tabular}

${ }^{\mathrm{y}} \mathrm{PCR}$ product was not polymorphic after restriction enzyme digestion and single strand conformation polymorphism (SSCP) analysis in either the Escort or in the Nikita family.

${ }^{\mathrm{z}}$ SSCP were not scorable in Escort and Nikita families. 


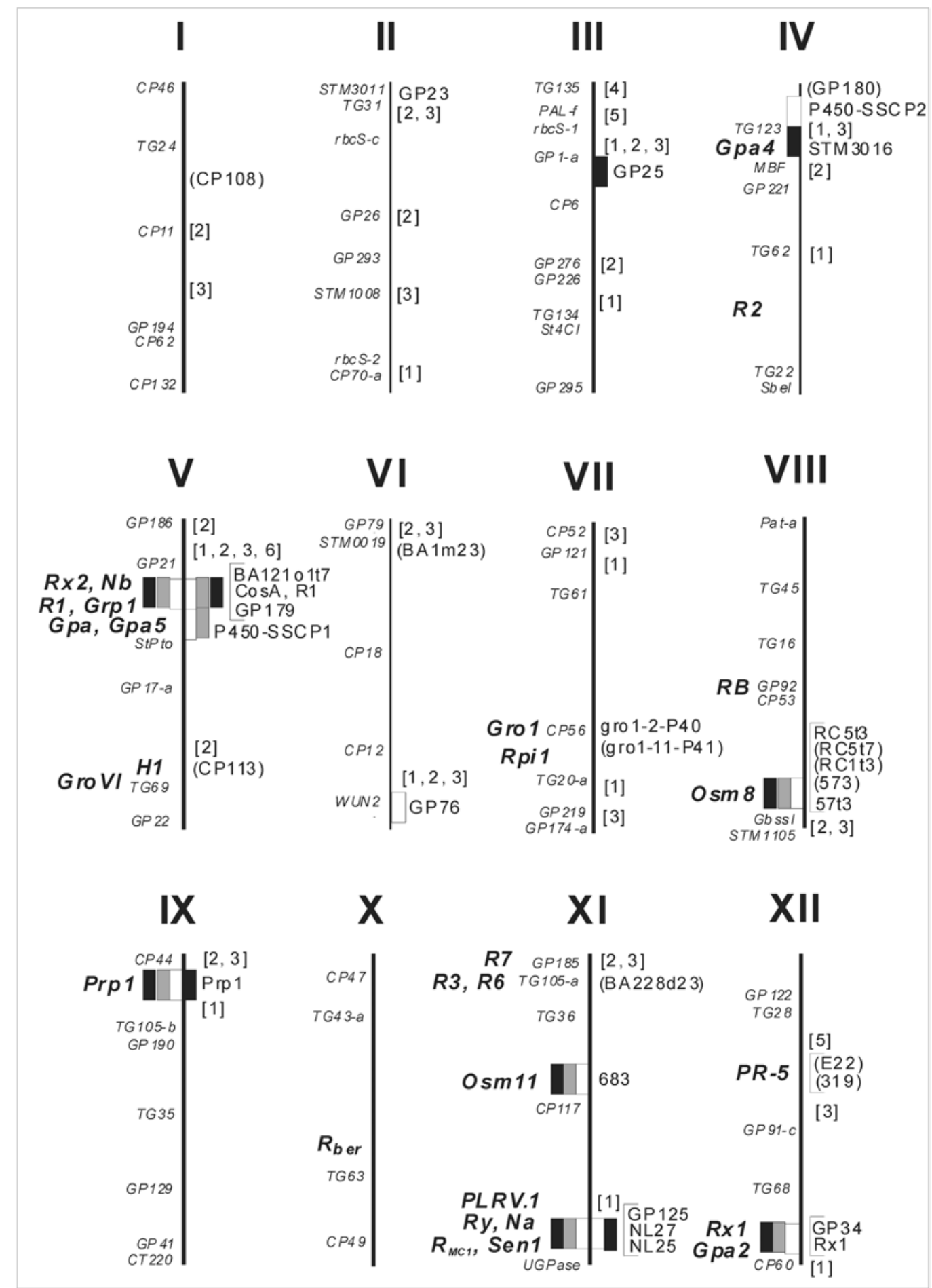

Fig. 2. Genomic positions of 30 polymerase chain reaction (PCR)-based markers and quantitative trait loci (QTL) for maturity, area under disease progress curve (AUDPC), and maturity-corrected resistance (MCR) detected in the tetraploid Escort and Nikita families. The 12 potato chromosomes are represented as schematic linkage groups with anchor restriction fragment length polymorphism and microsatellite loci at approximate positions on the left and the PCR markers tested on the right. PCR markers that did not segregate or were not scorable are shown in parentheses. Bars to the left and right of the linkage groups indicate a significant effect $(P \leq 0.05)$ of PCR marker alleles in the map segment on maturity (white bars), AUDPC (grey bars), and MCR (black bars), separately or in combination, in families Nikita and Escort, respectively. Map segments where QTL for late blight resistance have been located previously in diploid mapping populations are indicated to the right by numbers in brackets. $1=$ Leonards-Schippers et al. 1994, $2=$ Oberhagemann et al. 1999, $3=$ Collins et al. $1999,4=$ Ewing et al. 2000, $5=$ Trognitz et al. 2002, and $6=$ Visker et al. 2003. Positions of major genes for late blight resistance are shown on the left. $R 1=$ LeonardsSchippers et al. 1992, Ballvora et al. 2002; $R 3, R 6, R 7=$ El-Kharbotly et al. 1994,$1996 ; R 2=\mathrm{Li}$ et al. $1998 ; R_{\text {ber }}=$ Ewing et al. $2000 ; R B=$ Naess et al. 2000 , Song et al. 2003; and Rpil = Kuhl et al. 2001. Single genes or major QTL for resistance to other pathogens that are located in the map segments tagged by the PCR markers are: nematode resistance genes Grol (Barone et al. 1990), H1 (Gebhardt et al. 1993; Pineda et al. 1993), GroVI (Jacobs et al. 1996), Gpa2, Grp1, Gpa5 (Rouppe van der Voort et al. 1997, 1998, 2000), Gpa (Kreike et al. 1994), Gpa4 (Bradshaw et al. 1998), and $R_{\mathrm{Mcl}}$ (Brown et al. 1996); virus resistance genes Rx1, Rx2 (Ritter et al. 1991; Bendahmane et al. 1999, 2000), Nb (De Jong et al. 1997), Ry (Brigneti et al. 1997; Hämäläinen et al. 1997), Na (Hämäläinen et al. 2000), and PLRV.1 (Marczewski et al. 2001);and wart resistance gene Sen1 (Hehl et al. 1999). Pathogenesis-related loci tagged by the PCR markers were Prp1 (Leonards-Schippers et al. 1994), Osm8, Osm11, and PR-5 (Castillo-Ruiz 2002; Trognitz et al. 2002). 
ments in the Escort and Nikita families, respectively, were reproducibly linked to QTL, separately or in combination, for maturity, AUDPC, and MCR (Tables 3 and 4, Fig. 2). Seventeen marker alleles showed persistent effects in the same direction but of variable size over two or three years of testing, the average trait value over all years, or both. None of the marker alleles gave consistent results in both populations. However, markers tagging map segments $\mathrm{V}_{\mathrm{p}}, \mathrm{IX}_{\mathrm{sd}}$, and $\mathrm{XI}_{\mathrm{ld}}$ showed effects in both families, although the significant marker alleles only partially overlapped and effects varied across populations and years. No major effects were detected when the markers were tested individually. Fewer significant marker alleles and smaller effects were found in the Escort family as compared with the Nikita family. The amount of the variance explained by a single marker allele ranged from 4.4 to $11.6 \%$ in the Escort family and from 4.1 to $17.4 \%$ in the Nikita family. Of 15 significant marker alleles in the Nikita family, 11 were linked to higher susceptibility, earlier maturity, or both. In the Escort family, five marker alleles were linked to higher susceptibility, earlier maturity, or both and six were linked to higher resistance, later maturity, or both. All three parents contributed positive (more resistant, earlier maturing) and negative (more susceptible, later maturing) trait alleles. Interestingly, marker allele GP179-570 was linked to opposite effects in the two families. In Escort, GP179-570 was linked to higher susceptibility and in Nikita to higher resistance.

Effects on maturity were found in three of five map segments of the Escort family and in all seven map segments of the Nikita family. The largest and most consistent effects on maturity were observed in segments $\mathrm{V}_{\mathrm{p}}$ and $\mathrm{XI}_{\mathrm{ld}}$ of the Escort family (Table 3) and in segments $\mathrm{V}_{\mathrm{p}}, \mathrm{XI}_{\mathrm{p}}$, and $\mathrm{XI}_{\mathrm{ld}}$ of the Nikita family (Table 4). In the Escort family, effects on AUDPC were detected only in segment $V_{p}$ (Table 3), whereas in the Nikita family, effects on AUDPC were linked to markers in six segments, $\mathrm{V}_{\mathrm{p}}, \mathrm{VIII}_{\mathrm{ld}}, \mathrm{IX}_{\mathrm{sd}}, \mathrm{XI}_{\mathrm{p}}, \mathrm{XI}_{\mathrm{ld}}$, and $\mathrm{XII}_{\mathrm{ld}}$ (Table 4). Marker alleles were linked to MCR in the Escort family in four segments, $\mathrm{III}_{\mathrm{p}}, \mathrm{V}_{\mathrm{p}}, \mathrm{IX}_{\mathrm{sd}}$, and $\mathrm{XI}_{\mathrm{ld}}$, and in all segments in the Nikita family except segment IV $_{\text {sd. }}$. The most persistent effects on MCR (average trait values over two or three years) were associated with markers tagging segments $\mathrm{V}_{\mathrm{p}}, \mathrm{IX}_{\mathrm{sd}}$, and $\mathrm{XI}_{\mathrm{ld}}$ in both families and, in addition, with markers tagging segments $\mathrm{VIII}_{\mathrm{ld}}, \mathrm{XI}_{\mathrm{p}}$, and $\mathrm{XII}_{\mathrm{ld}}$ in the Nikita family. The largest effects on MCR were detected by marker

Table 2. Observed segregation, genetic model and origin of marker alleles, which detected effects on either late blight resistance, plant maturity, or both either individually, in interaction tests, or both

\begin{tabular}{|c|c|c|c|c|c|c|c|c|c|}
\hline \multirow[b]{3}{*}{$\mathbf{M S}^{\mathbf{x}}$} & \multirow[b]{3}{*}{ Marker allele } & \multicolumn{4}{|c|}{ Escort $\times$ Leyla } & \multicolumn{4}{|c|}{ Nikita $\times$ Leyla } \\
\hline & & \multicolumn{3}{|c|}{ Allele segregation ratio } & \multirow[b]{2}{*}{ Origin of allele } & \multicolumn{3}{|c|}{ Allele segregation ratio } & \multirow[b]{2}{*}{ Origin of allele } \\
\hline & & Present & Absent & Best model & & Present & Absent & Best model & \\
\hline \multirow[t]{4}{*}{$\mathrm{II}_{\mathrm{sd}}$} & GP23-SSCP1 & 47 & 36 & $1: 1$ & Leyla & 86 & 9 & $11: 1$ & Nikita, Leyla \\
\hline & GP23-SSCP2 & 49 & 34 & $3: 1_{\text {distorted }}$ & Escort, Leyla & 62 & 33 & $3: 1_{\text {distorted }}$ & Nikita, Leyla \\
\hline & GP23-SSCP4 & 41 & 42 & $1: 1$ & Leyla & 49 & 46 & $1: 1$ & Leyla \\
\hline & GP23-SSCP5 & 65 & 18 & $5: 1$ & Escort & 95 & 0 & $-^{\mathrm{y}}$ & Nikita \\
\hline \multirow[t]{3}{*}{$\mathrm{III}_{\mathrm{p}}$} & GP25-SSCP1 & 70 & 14 & $5: 1$ & Leyla & 78 & 17 & $5: 1$ & Leyla \\
\hline & GP25-SSCP2 & 36 & 48 & $1: 1_{\text {distorted }}$ & Escort & 51 & 44 & $1: 1$ & Nikita \\
\hline & GP25-TaqI-550 & 37 & 43 & $1: 1$ & Escort & - & - & - & Absent \\
\hline \multirow[t]{2}{*}{$\mathrm{IV}_{\mathrm{sd}}$} & P450-SSCP2 & 64 & 20 & $3: 1$ & Escort, Leyla & 77 & 18 & $3: 1$ & Nikita, Leyla \\
\hline & STM3016-151 & - & - & - & Absent & 47 & 48 & $1: 1$ & Nikita \\
\hline \multirow[t]{11}{*}{$\mathrm{V}_{\mathrm{p}}$} & $\operatorname{Cos} \mathrm{A}-210^{\mathrm{z}}$ & 71 & 13 & $5: 1$ & Escort & 34 & 61 & $1: 1_{\text {distorted }}$ & Nikita \\
\hline & $\mathrm{R} 1-1400^{\mathrm{z}}$ & 73 & 12 & $5: 1$ & Escort & 33 & 62 & $1: 1_{\text {distorted }}$ & Nikita \\
\hline & GP179-570 & 35 & 49 & $1: 1_{\text {distorted }}$ & Escort & 42 & 53 & $1: 1$ & Nikita \\
\hline & BA121o1t7-SSCP1 & 57 & 27 & $3: 1$ & Escort, Leyla & 83 & 12 & $3: 1_{\text {distorted }}$ & Nikita, Leyla \\
\hline & BA121o1t7-SSCP2 & 70 & 13 & $3: 1_{\text {distorted }}$ & Escort, Leyla & 79 & 16 & $3: 1_{\text {distorted }}$ & Nikita, Leyla \\
\hline & BA121o1t7-SSCP3 & 34 & 50 & $1: 1_{\text {distorted }}$ & Leyla & 47 & 48 & $3: 1_{\text {distorted }}$ & Nikita, Leyla \\
\hline & BA121o1t7-SSCP4 & 72 & 11 & $11: 1$ & Escort, Leyla & 74 & 19 & $3: 1$ & Nikita, Leyla \\
\hline & BA121o1t7-SSCP5b & 41 & 43 & $1: 1$ & Escort & - & - & - & Absent \\
\hline & BA121o1t7-SSCP6a & - & - & - & Absent & 41 & 52 & $1: 1$ & Nikita \\
\hline & BA121o1t7-SSCP9 & 60 & 23 & $1: 1_{\text {distorted }}$ & Leyla & 75 & 20 & $3: 1$ & Nikita, Leyla \\
\hline & P450-SSCP1 & 46 & 38 & $1: 1$ & Escort & 91 & 4 & $-^{\mathrm{y}}$ & Nikita \\
\hline \multirow[t]{2}{*}{$\mathrm{VI}_{\mathrm{ld}}$} & GP76-Rsa-500 & 60 & 24 & $5: 1_{\text {distorted }}$ & Escort & - & - & - & Absent \\
\hline & GP76-Rsa-600 & 68 & 16 & $3: 1$ & Escort, Leyla & 94 & 0 & $-^{\mathrm{y}}$ & Nikita, Leyla \\
\hline $\mathrm{VII}_{\mathrm{p}}$ & gro1-2-P40-500 & - & - & - & Absent & 75 & 20 & $5: 1$ & Nikita \\
\hline \multirow[t]{2}{*}{$\mathrm{VIII}_{\mathrm{ld}}$} & $57 \mathrm{t} 3-\mathrm{SSCP}$ & 32 & 47 & $1: 1_{\text {distorted }}$ & Escort & 74 & 20 & $5: 1$ & Nikita \\
\hline & RC5t3-RsaI-280 & - & - & - & Absent & 51 & 44 & $1: 1$ & Nikita \\
\hline $\mathrm{IX}_{\mathrm{sd}}$ & Prp1-200 & 41 & 43 & $1: 1$ & Leyla & 70 & 25 & $3: 1$ & Nikita, Leyla \\
\hline \multirow[t]{2}{*}{$\mathrm{XI}_{\mathrm{p}}$} & 683-SSCP1 & 37 & 41 & $1: 1$ & Escort & 49 & 42 & $1: 1$ & Nikita \\
\hline & 683-SSCP2 & 69 & 9 & $5: 1$ & Leyla & 75 & 17 & $5: 1$ & Leyla \\
\hline \multirow[t]{8}{*}{$\mathrm{XI}_{\mathrm{ld}}$} & GP125-SSCP1 & - & - & - & Absent & 72 & 22 & $5: 1_{\text {distorted }}$ & Nikita \\
\hline & GP125-SSCP2 & 80 & 2 & $-^{\mathrm{y}}$ & Escort, Leyla & 51 & 43 & $3: 1_{\text {distorted }}$ & Nikita, Leyla \\
\hline & GP125-SSCP4 & - & - & - & Absent & 40 & 55 & $1: 1$ & Nikita \\
\hline & NL25-SSCP3 & 62 & 17 & $3: 1$ & Escort, Leyla & 69 & 24 & $3: 1$ & Nikita, Leyla \\
\hline & NL27-SSCP3 & 63 & 11 & $5: 1$ & Leyla & 79 & 14 & $5: 1$ & Leyla \\
\hline & NL27-SSCP4b & 41 & 35 & $1: 1$ & Leyla & 17 & 76 & $1: 1_{\text {distorted }}$ & Leyla \\
\hline & NL27-SSCP5 & - & - & - & Absent & 45 & 48 & $1: 1$ & Nikita \\
\hline & NL27-SSCP7 & 36 & 41 & $1: 1$ & Escort & 72 & 21 & $5: 1_{\text {distorted }}$ & Nikita \\
\hline \multirow{3}{*}{$\mathrm{XII}_{\mathrm{ld}}$} & GP34-SSCP2 & 46 & 35 & $1: 1$ & Leyla & 85 & 10 & $11: 1$ & Nikita, Leyla \\
\hline & Rx1-SSCP1 & 95 & 0 & $-^{y}$ & Escort, Leyla & 43 & 41 & $1: 1$ & Leyla \\
\hline & Rx1-SSCP2 & - & - & - & Absent & 32 & 52 & $1: 1_{\text {distorted }}$ & Nikita \\
\hline
\end{tabular}


Prp1-200 (IX IX $\left._{\mathrm{sd}}\right)$ in the Escort family and by markers R1-1400 $\left(\mathrm{V}_{\mathrm{p}}\right)$, GP125-SSCP1 $\left(\mathrm{XI}_{\mathrm{ld}}\right)$, and GP34-SSCP2 $\left(\mathrm{XII}_{\mathrm{ld}}\right)$ in the Nikita family.

All marker alleles were tested for pair-wise interactions, using as trait the all year average of MCR. Of 470 interaction tests in the Escort family, eight $(1.7 \%)$ were significant at $P \leq$ 0.01, including two intralocus interactions (Table 5). Half of those interactions are expected to be chance effects. One interaction test was significant at $P \leq 0.001$ (Table 5). In the Nikita family, 22 of 579 interaction tests $(3.8 \%)$ were significant at $P$ $\leq 0.01$, with five to six of those expected by chance. Eight interaction tests were significant at $P \leq 0.001$ (Table 6). No intralocus interaction was found in the Nikita family. Testing for interaction identified two additional map segments that were not significant in the single marker tests. These were map segments $\mathrm{II}_{\mathrm{sd}}$, tagged by GP23 alleles in the Escort family (Table 5), and map segment $\mathrm{VII}_{\mathrm{p}}$, tagged by the marker gro1-
2-P40 in the Nikita family (Table 6). Markers tagging map segment $\mathrm{V}_{\mathrm{p}}$ in both families interacted with unlinked loci on chromosomes III, IV, VII, VIII, IX, XI, and XII. The marker allele gro1-2-P40-500 ( $\left.\mathrm{VII}_{\mathrm{p}}\right)$ interacted with five other loci. Single interlocus interactions explained between 9 and $24 \%$ of the phenotypic variance. Genotypes having favorable allele combinations (negative MCR class means) were less frequent than genotypes with unfavorable allele combinations (Tables 5 and 6).

Multiple regression analysis with forward selection was performed with all marker alleles for the all-year average of MCR in families Escort and Nikita (Table 7). With exception of GP179-570, the selected marker alleles differed between the families. For the Nikita family, five marker alleles tagging segments $\mathrm{V}_{\mathrm{p}}, \mathrm{VIII}_{\mathrm{ld}}, \mathrm{XI}_{\mathrm{p}}$, and $\mathrm{XII}_{\mathrm{ld}}$ were selected that together explained $38 \%$ of the phenotypic variance. Selection for presence of the marker alleles in segment $V_{p}$ and absence of the

Table 3. Effects of single marker alleles on maturity, AUDPC (area under disease progress curve), and MCR (maturity-corrected resistance) in population Escort $\times$ Leyla. The phenotypic variance explained by the marker $\left(R^{2}\right)$ is shown as percentage

\begin{tabular}{|c|c|c|c|c|c|c|c|c|c|c|c|c|}
\hline \multirow[b]{2}{*}{$\mathbf{M S}^{\mathbf{w}}$} & \multirow[b]{2}{*}{ Marker allele } & \multirow[b]{2}{*}{ Origin of allele } & \multicolumn{4}{|c|}{ Maturity } & \multicolumn{3}{|c|}{ AUDPC } & \multicolumn{3}{|c|}{ MCR } \\
\hline & & & 1999 & 2000 & 2001 & $\begin{array}{l}1999- \\
2001\end{array}$ & 2000 & 2001 & $\begin{array}{l}2000- \\
2001\end{array}$ & 2000 & 2001 & $\begin{array}{l}2000- \\
2001\end{array}$ \\
\hline $\mathrm{III}_{\mathrm{p}}$ & GP25-SSCP1 $1^{x}$ & Leyla & $-^{\mathrm{y}}$ & - & - & - & - & - & - & - & $7.5^{* *}$ & - \\
\hline \multirow{5}{*}{$V_{p}^{p}$} & $\operatorname{Cos} \mathrm{A}-210^{\mathrm{x}}$ & Escort & - & - & - & - & - & $7.5^{* *}$ & $5.6^{*}$ & - & $4.9^{*}$ & $4.4^{*}$ \\
\hline & GP179-570 ${ }^{z}$ & Escort & _- & - & - & - & $5.5^{*}$ & $4.7 *$ & $6.5^{*}$ & - & $4.9 *$ & $5.7 *$ \\
\hline & BA12101t7-SSCP $3^{x}$ & Leyla & - & $7.4 * *$ & $7.2 * *$ & $7.6 * *$ & - & $6.8 *$ & - & - & - & - \\
\hline & BA121o1t7-SSCP5b & Escort & $5.3^{*}$ & $6.6^{*}$ & - & $5.1^{*}$ & $7.8 * *$ & $5.9 *$ & $8.8^{* *}$ & - & $4.6^{*}$ & $5.2^{*}$ \\
\hline & $\mathrm{P} 450-\mathrm{SSCP}^{\mathrm{x}}$ & Escort & $7.6^{* * *}$ & $8.1 * *$ & $7.2 * *$ & $9.8 * *$ & $5.7 *$ & $6.8 *$ & $7.6^{* * *}$ & - & - & - \\
\hline $\mathrm{VI}_{\mathrm{ld}}$ & GP76-Rsa-600 & Escort, Leyla & $4.8^{*}$ & - & $5.4 *$ & $5.7 *$ & - & - & - & - & - & - \\
\hline $\mathrm{IX}_{\mathrm{sd}}$ & $\operatorname{Prp} 1-200^{\mathrm{z}}$ & Leyla & - & - & - & - & - & _- & - & $6.0 *$ & - & $7.7 * *$ \\
\hline \multirow{3}{*}{$\mathrm{XI}_{\mathrm{ld}}$} & NL25-SSCP $3^{\mathrm{x}}$ & Escort, Leyla & - & $6.9^{*}$ & $11.6^{* *}$ & $7.8 * *$ & - & - & - & - & - & - \\
\hline & NL27-SSCP3 ${ }^{\mathrm{Z}}$ & Leyla & _- & - & $5.9^{*}$ & - & - & _- & _- & _- & $7.8^{*}$ & _- \\
\hline & NL27-SSCP4b & Leyla & - & - & - & - & - & - & - & $5.2 *$ & - & $5.4^{*}$ \\
\hline
\end{tabular}

${ }^{\mathrm{w}}$ The map segment (MS) tagged by the markers is indicated by chromosome number and suffixes $\mathrm{p}$, ld, and sd for proximal or distal position on the long and short chromosome arm, respectively (Dong et al. 2000).

${ }^{x}$ Marker allele present $=$ plant is more resistant and/or later maturing

y - indicates $P>0.05, *$ indicates $P \leq 0.05$, and ** indicates $P \leq 0.01$.

${ }^{\mathrm{z}}$ Marker allele present $=$ plant is more susceptible and/or earlier maturing

Table 4. Effects of single marker alleles on maturity, AUDPC (area under disease progress curve), and MCR (maturity-corrected resistance) in population Nikita $\times$ Leyla. The phenotypic variance explained by the marker $\left(\mathrm{R}^{2}\right)$ is shown as percentage.

\begin{tabular}{|c|c|c|c|c|c|c|c|c|c|c|c|c|c|c|c|}
\hline \multirow[b]{2}{*}{$\mathbf{M S}^{\mathbf{w}}$} & \multirow[b]{2}{*}{$\begin{array}{c}\text { Marker } \\
\text { allele }\end{array}$} & \multirow[b]{2}{*}{$\begin{array}{l}\text { Origin of } \\
\text { allele }\end{array}$} & \multicolumn{5}{|c|}{ Maturity } & \multicolumn{4}{|c|}{ AUDPC } & \multicolumn{4}{|c|}{ MCR } \\
\hline & & & 1999 & 2000 & 2001 & 2002 & $\begin{array}{l}1999- \\
2002\end{array}$ & 2000 & 2001 & 2002 & $\begin{array}{l}2000- \\
2002\end{array}$ & 2000 & 2001 & 2002 & $\begin{array}{l}2000- \\
2002\end{array}$ \\
\hline$\overline{\mathrm{IV}_{\mathrm{sd}}}$ & $\mathrm{P} 450-\mathrm{SSCP}^{\mathrm{x}}$ & Nikita, Leyla & $4.2 *$ & $-^{y}$ & - & - & $4.9^{*}$ & - & - & - & - & - & - & - & - \\
\hline \multirow[t]{4}{*}{$\mathrm{V}_{\mathrm{p}}$} & $\mathrm{R} 1-1400^{\mathrm{z}}$ & Nikita & - & - & $10.9 \S$ & $17.4 \S \S$ & $7.7 * *$ & - & $9.1 * *$ & $7.5^{*}$ & $7.7 *$ & - & $11.9 \S$ & $8.0^{* * *}$ & $8.7 * *$ \\
\hline & GP179-570 & Nikita & - & - & - & - & - & $4.8^{*}$ & $4.2^{*}$ & - & $4.0^{*}$ & $4.4^{*}$ & - & - & - \\
\hline & $\begin{array}{l}\text { SSCP3 }^{\mathrm{z}} \\
\text { BA121o1t7- }\end{array}$ & Nikita, Leyla & $4.6^{*}$ & - & - & - & - & - & - & - & - & - & - & - & - \\
\hline & SSCP4 $^{x}$ & Nikita, Leyla & $4.6^{*}$ & $4.4 *$ & $5.8^{*}$ & $5.8^{*}$ & $7.4^{* *}$ & - & - & - & - & - & - & - & - \\
\hline $\mathrm{VIII}_{\mathrm{ld}}$ & $57 \mathrm{~T} 3-\mathrm{SSCP}^{\mathrm{x}}$ & Nikita & _- & - & - & $4.9 *$ & - & _- & $4.2^{*}$ & $5.5^{*}$ & $4.7 *$ & _- & - & $6.0 *$ & $5.5^{*}$ \\
\hline $\mathrm{IX}_{\mathrm{sd}}$ & $\operatorname{Prp} 1-200^{x}$ & Nikita, Leyla & - & - & $4.5^{*}$ & $6.5^{* *}$ & $4.1 *$ & - & $5.5^{*}$ & $5.6^{*}$ & $4.4^{*}$ & - & $8.7^{* *}$ & $6.9 * *$ & $5.6^{*}$ \\
\hline \multirow[t]{2}{*}{$\mathrm{XI}_{\mathrm{p}}$} & $683-S S C P 1^{x}$ & Nikita & - & $8.0^{* *}$ & $7.0^{* *}$ & $5.6^{*}$ & $8.6^{* *}$ & $4.7 *$ & - & $5.4^{*}$ & $6.4^{*}$ & - & - & - & - \\
\hline & $683-\mathrm{SSCP}^{\mathrm{x}}$ & Leyla & - & - & - & - & - & - & - & - & - & - & $6.4^{*}$ & - & $4.1 *$ \\
\hline \multirow[t]{6}{*}{$\mathrm{XI}_{\mathrm{ld}}$} & $\begin{array}{l}\text { GP125- } \\
\text { SSCP1x }\end{array}$ & Nikita & - & $\ldots$ & $83 * *$ & 1258 & $86 * *$ & $5.4 *$ & $85 * *$ & $86 * *$ & $98 * *$ & $\ldots$ & $83 * *$ & $85 * *$ & $83 * *$ \\
\hline & GP125- & N⿴囗⿰丿㇄丄а & - & - & 0.5 & $12.0 \mathrm{~g}$ & 0.0 & $J .4$ & 0.0 & 0.0 & 9.0 & - & 0.5 & 0.5 & 0.5 \\
\hline & $\mathrm{SSCP}^{\mathrm{z}}$ & Nikita, Leyla & - & - & $7.5^{* *}$ & $5.4^{*}$ & - & - & - & $4.2^{*}$ & - & - & - & $4.1^{*}$ & - \\
\hline & NL25-SSCP $3^{x}$ & Nikita, Leyla & - & - & - & - & - & - & $4.3^{*}$ & - & - & - & - & - & - \\
\hline & NL27-SSCP $3^{\mathrm{x}}$ & Leyla & - & _- & - & - & - & _- & $6.4 * *$ & - & - & _- & $4.8^{*}$ & - & - \\
\hline & NL27-SSCP5 ${ }^{\mathrm{x}}$ & Nikita & - & - & $8.1^{* *}$ & $4.5^{*}$ & $6.2^{*}$ & $4.1 *$ & $4.5^{*}$ & $5.8^{*}$ & $6.5^{* *}$ & - & - & $4.2^{*}$ & - \\
\hline $\mathrm{XII}_{\mathrm{ld}}$ & GP34-SSCP2 $2^{x}$ & Nikita, Leyla & - & - & - & $4.3^{*}$ & - & - & $4.5^{*}$ & $6.6^{* *}$ & $4.5^{*}$ & - & $12.2 \S$ & $10.1 * *$ & $10.4 * *$ \\
\hline
\end{tabular}

${ }^{\mathrm{w}}$ The map segment (MS) tagged by the markers is indicated by chromosome number and suffixes $\mathrm{p}$, ld, and sd for proximal or distal position on the long and short chromosome arm, respectively (Dong et al. 2000).

${ }^{\mathrm{x}}$ Marker allele present $=$ plant is more susceptible, earlier maturing, or both.

${ }^{y}-$ indicates $P>0.05$, * indicates $P \leq 0.05$, ** indicates $P \leq 0.01$, $\S$ indicates $P \leq 0.001$, and $\S \S$ indicates $P \leq 0.0001$.

${ }^{\mathrm{z}}$ Marker allele present $=$ plant is more resistant, later maturing, or both. 
marker alleles in the other segments should improve resistance. Taking into account the segregation ratio of each marker allele (Table 2) and assuming independent segregation of each marker allele, the expected frequency of genotypes that combine the positive alleles of the five markers suggested by the model was 0.0008 or eight in 10,000 plants. Only two markers in segments $\mathrm{V}_{\mathrm{p}}$ and $\mathrm{XI}_{\mathrm{ld}}$ were selected in the Escort family that together explained $15 \%$ of the phenotypic variance. Selection against the GP179-570 and NL27-SSCP4b marker alleles should increase resistance. The expected frequency of genotypes lacking both the GP179-570 and NL27-SSCP4b allele was 0.27 or 27 of 100 plants.

Table 5. Interactions $(P \leq 0.01)$ between pairs of marker alleles in population Escort $\times$ Leyla for the two-year average of MCR (maturity-corrected resistance). The phenotypic variance explained by the marker $\left(R^{2}\right)$ is shown as percentage.

\begin{tabular}{|c|c|c|c|c|c|c|c|c|c|c|c|c|}
\hline \multirow[b]{3}{*}{$\operatorname{MS~} 1^{x}$} & \multirow[b]{3}{*}{ Marker allele 1} & \multirow[b]{3}{*}{ MS 2} & \multirow[b]{3}{*}{ Marker allele 2} & \multicolumn{8}{|c|}{ Marker class ${ }^{\mathrm{w}}$} & \multirow[b]{3}{*}{$\mathbf{R}^{2 \mathrm{z}}$} \\
\hline & & & & \multicolumn{2}{|c|}{00} & \multicolumn{2}{|c|}{02} & \multicolumn{2}{|c|}{10} & \multicolumn{2}{|c|}{12} & \\
\hline & & & & Mean $^{y}$ & $\mathbf{n}$ & Mean & $\mathbf{n}$ & Mean & $\mathbf{n}$ & Mean & $\bar{n}$ & \\
\hline \multirow[t]{4}{*}{$\mathrm{II}_{\mathrm{sd}}$} & GP23-SSCP1 & $\mathrm{V}_{\mathrm{p}}$ & P450-SSCP1 & $13.3 \mathrm{a}$ & 17 & $-8.2 \mathrm{~b}$ & 19 & $4.1 \mathrm{ab}$ & 21 & $8.0 \mathrm{a}$ & 26 & $13.1^{* *}$ \\
\hline & GP23-SSCP1 & $\mathrm{VI}_{\mathrm{ld}}$ & GP76-RsaI-500 & $-19.0 \mathrm{a}$ & 7 & $7.0 \mathrm{~b}$ & 29 & $11.9 \mathrm{~b}$ & 17 & $3.1 \mathrm{~b}$ & 30 & $14.0^{* * * *}$ \\
\hline & GP23-SSCP2 & $\mathrm{VI}_{\mathrm{ld}}$ & GP76-RsaI-500 & $-16.4 \mathrm{a}$ & 6 & $7.1 \mathrm{~b}$ & 28 & $9.3 \mathrm{~b}$ & 18 & $3.1 \mathrm{ab}$ & 31 & $9.2 * *$ \\
\hline & GP23-SSCP4 & $\mathrm{II}_{\mathrm{sd}}$ & GP23-SSCP5 & $-14.6 \mathrm{a}$ & 7 & $7.8 \mathrm{~b}$ & 35 & $10.2 \mathrm{~b}$ & 11 & $2.7 \mathrm{ab}$ & 30 & $9.4 * *$ \\
\hline \multirow[t]{5}{*}{$\mathrm{V}_{\mathrm{p}}$} & BA121o1t7-SSCP1 & $\mathrm{IX}_{\mathrm{sd}}$ & Prp1-210 & $13.9 \mathrm{a}$ & 14 & $7.5 \mathrm{ab}$ & 13 & $-7.4 \mathrm{~b}$ & 30 & $10.1 \mathrm{a}$ & 28 & $17.5^{* *}$ \\
\hline & BA121o1t7-SSCP1 & $\mathrm{XI}_{\mathrm{ld}}$ & NL25-SSCP3 & $30.4 \mathrm{a}$ & 6 & $4.6 \mathrm{~b}$ & 19 & $-0.8 \mathrm{~b}$ & 11 & $2.6 \mathrm{~b}$ & 43 & $13.6^{* *}$ \\
\hline & BA121o1t7-SSCP9 & $\mathrm{III}_{\mathrm{p}}$ & GP25-TaqI-550 & $18.8 \mathrm{a}$ & 12 & $-3.2 \mathrm{~b}$ & 11 & $0.6 \mathrm{~b}$ & 31 & $3.3 \mathrm{ab}$ & 24 & $11.9 * *$ \\
\hline & BA121o1t7-SSCP9 & $\mathrm{IX}_{\mathrm{sd}}$ & Prp1-210 & $14.8 \mathrm{a}$ & 11 & $4.9 \mathrm{ab}$ & 12 & $-6.3 b$ & 31 & $11.8 \mathrm{a}$ & 29 & $17.5^{* *}$ \\
\hline & BA121o1t7-SSCP2 & $\mathrm{V}_{\mathrm{p}}$ & BA121o1t7-SSCP4 & $-5.1 \mathrm{a}$ & 4 & $22.0 \mathrm{~b}$ & 9 & $12.3 \mathrm{ab}$ & 7 & $0.6 \mathrm{a}$ & 62 & $12.9^{* *}$ \\
\hline
\end{tabular}

${ }^{\mathrm{w}}$ Mean MCR and number of individuals $\mathrm{n}$ that have neither of the two marker alleles (00), only marker allele 2 (02), only marker allele 1 (10), or both marker alleles (12). Negative means indicate increased resistance.

${ }^{\mathrm{x}}$ The map segment (MS) tagged by the markers is indicated by chromosome number and suffixes $\mathrm{p}$, ld, and sd for proximal or distal position on the long and short arm, respectively (Dong et al. 2000).

${ }^{y}$ Groups of individuals labeled with different small letters (a or b) are different $(P \leq 0.05)$ from each other.

z ** indicates $P \leq 0.01, * * *$ indicates $P \leq 0.001$.

Table 6. Interactions $(P<0.01)$ between pairs of marker alleles in population Nikita $\times$ Leyla for the three-year average of MCR (maturity-corrected resistance). The phenotypic variance explained by the marker $\left(\mathrm{R}^{2}\right)$ is shown as percentage.

\begin{tabular}{|c|c|c|c|c|c|c|c|c|c|c|c|c|}
\hline \multirow[b]{3}{*}{ MS $1^{x}$} & \multirow[b]{3}{*}{ Marker allele 1} & \multirow[b]{3}{*}{ MS 2} & \multirow[b]{3}{*}{ Marker allele 2} & \multicolumn{8}{|c|}{ Marker class $^{w}$} & \multirow[b]{3}{*}{$\mathbf{R}^{2 z}$} \\
\hline & & & & \multicolumn{2}{|c|}{00} & \multicolumn{2}{|l|}{02} & \multicolumn{2}{|l|}{10} & \multicolumn{2}{|c|}{12} & \\
\hline & & & & $\operatorname{Mean}^{y}$ & $\mathbf{n}$ & Mean & $\mathbf{n}$ & Mean & $\mathbf{n}$ & Mean & $\mathbf{n}$ & \\
\hline \multirow[t]{2}{*}{$\mathrm{III}_{\mathrm{p}}$} & GP25-SSCP2 & $\mathrm{V}_{\mathrm{p}}$ & BA121o1t7-SSCP2 & $12.3 \mathrm{~b}$ & 5 & $1.1 \mathrm{ab}$ & 39 & $-9.9 \mathrm{a}$ & 11 & $10.7 \mathrm{~b}$ & 40 & $10.2 * *$ \\
\hline & GP25-SSCP2 & $\mathrm{XI}_{\mathrm{ld}}$ & GP125-SSCP2 & $0.8 \mathrm{ab}$ & 21 & $3.8 \mathrm{ab}$ & 23 & $16.8 \mathrm{a}$ & 22 & $-1.6 \mathrm{~b}$ & 28 & $10.5^{* *}$ \\
\hline \multirow{4}{*}{$\mathrm{IV}_{\mathrm{sd}}$} & STM3016-151 & $\mathrm{V}_{\mathrm{p}}$ & BA121o1t7-SSCP3 & $-10.3 \mathrm{a}$ & 19 & $8.1 \mathrm{~b}$ & 29 & $10.3 \mathrm{~b}$ & 29 & $4.7 \mathrm{ab}$ & 18 & $12.3^{* *}$ \\
\hline & STM3016-151 & $\mathrm{VII}_{\mathrm{p}}$ & gro1-2-P40-500 & $-10.3 \mathrm{a}$ & 14 & $5.4 \mathrm{ab}$ & 34 & $21.9 \mathrm{~b}$ & 6 & $6.2 \mathrm{ab}$ & 41 & $11.3^{* *}$ \\
\hline & STM3016-151 & $\mathrm{XI}_{\mathrm{ld}}$ & GP125-SSCP1 & $-22.9 \mathrm{a}$ & 12 & $9.2 \mathrm{~b}$ & 35 & $12.6 \mathrm{~b}$ & 10 & $7.0 \mathrm{~b}$ & 37 & $24.0 \S \S$ \\
\hline & STM3016-151 & $\mathrm{XI}_{\mathrm{ld}}$ & GP125-SSCP4 & $-8.6 \mathrm{a}$ & 27 & $12.9 \mathrm{~b}$ & 21 & $10.4 \mathrm{~b}$ & 28 & $4.8 \mathrm{ab}$ & 19 & $15.9 * *$ \\
\hline \multirow{11}{*}{$\mathrm{V}_{\mathrm{p}}$} & $\mathrm{R} 1-1400$ & $\mathrm{IV}_{\mathrm{sd}}$ & STM3016-151 & $9.0 \mathrm{a}$ & 32 & $9.2 \mathrm{a}$ & 30 & $-15.6 \mathrm{~b}$ & 16 & $6.4 \mathrm{ab}$ & 17 & $17.6 * *$ \\
\hline & R1-1400 & $\mathrm{III}_{\mathrm{p}}$ & GP25-SSCP1 & $25.0 \mathrm{a}$ & 11 & $5.6 \mathrm{~b}$ & 51 & $-14.2 b$ & 6 & $-2.1 \mathrm{~b}$ & 27 & $17.9 * *$ \\
\hline & $\mathrm{R} 1-1400$ & $\mathrm{VII}_{\mathrm{p}}$ & gro1-2-P40-500 & $16.2 \mathrm{a}$ & 12 & $7.4 \mathrm{a}$ & 50 & $-25.9 b$ & 8 & $2.6 \mathrm{a}$ & 25 & $21.5^{* * *}$ \\
\hline & R1-1400 & $\mathrm{VIII}_{\mathrm{ld}}$ & RC5T3-RsaI-280 & $11.5 \mathrm{a}$ & 28 & $7.2 \mathrm{a}$ & 34 & $-14.7 \mathrm{~b}$ & 16 & $5.5 \mathrm{a}$ & 17 & $16.9^{* *}$ \\
\hline & BA121o1t7-SSCP2 & $\mathrm{IX}_{\mathrm{sd}}$ & Prp1-210 & $-19.7 \mathrm{a}$ & 8 & $13.8 \mathrm{~b}$ & 8 & $3.2 \mathrm{~b}$ & 17 & $6.7 \mathrm{~b}$ & 62 & $12.8 * *$ \\
\hline & BA121o1t7-SSCP2 & $\mathrm{XI}_{\mathrm{ld}}$ & GP125-SSCP1 & $-38.1 \mathrm{a}$ & 4 & $10.1 \mathrm{~b}$ & 11 & $0.1 \mathrm{~b}$ & 18 & $7.7 \mathrm{~b}$ & 61 & $19.2 * *$ \\
\hline & BA121o1t7-SSCP2 & $\mathrm{XI}_{\mathrm{ld}}$ & GP125-SSCP4 & $-19.3 \mathrm{a}$ & 9 & $18.2 \mathrm{~b}$ & 7 & $5.1 \mathrm{~b}$ & 46 & $7.1 \mathrm{~b}$ & 33 & $15.0 * *$ \\
\hline & BA121o1t7-SSCP2 & $\mathrm{XI}_{1 \mathrm{~d}}$ & NL27-SSCP4b & $9.8 \mathrm{a}$ & 9 & $-19.4 \mathrm{~b}$ & 7 & $4.1 \mathrm{a}$ & 67 & $17.8 \mathrm{a}$ & 10 & $13.7^{* * *}$ \\
\hline & BA121o1t7-SSCP2 & $X I_{l d}$ & NL27-SSCP7 & $-15.8 \mathrm{a}$ & 7 & $7.0 \mathrm{ab}$ & 9 & $13.6 \mathrm{~b}$ & 14 & $4.1 \mathrm{ab}$ & 63 & $9.3^{* *}$ \\
\hline & BA121o1t7-SSCP2 & $\mathrm{XII}_{\mathrm{ld}}$ & GP34-SSCP2 & $-45.5 \mathrm{a}$ & 4 & $11.1 \mathrm{~b}$ & 12 & $3.8 \mathrm{~b}$ & 6 & $6.1 \mathrm{~b}$ & 73 & $24.0^{* * * *}$ \\
\hline & ВA121o1t7-SSCP6a & $\mathrm{VIII}_{\mathrm{dd}}$ & RC5T3-RsaI-280 & $-3.7 \mathrm{a}$ & 24 & $13.7 \mathrm{~b}$ & 28 & $9.5 \mathrm{ab}$ & 18 & $-3.2 \mathrm{a}$ & 21 & $13.0^{* * * *}$ \\
\hline \multirow[t]{7}{*}{$\mathrm{VII}_{\mathrm{p}}$} & gro1-2-P40-500 & $\mathrm{V}_{\mathrm{p}}$ & BA121o1t7-SSCP3 & $-20.9 \mathrm{a}$ & 10 & $19.5 \mathrm{~b}$ & 10 & $8.2 \mathrm{~b}$ & 38 & $3.3 \mathrm{~b}$ & 37 & $20.8 \S \S$ \\
\hline & gro1-2-P40-500 & $\mathrm{IV}_{\mathrm{sd}}^{\mathrm{P}}$ & P450-SSCP2 & $-24.9 \mathrm{a}$ & 4 & $5.4 \mathrm{~b}$ & 16 & $10.5 \mathrm{~b}$ & 14 & $4.7 \mathrm{~b}$ & 61 & $8.9 * *$ \\
\hline & gro1-2-P40-500 & $\mathrm{VIII}_{\mathrm{ld}}$ & 57T3-SSCP & $-30.8 \mathrm{a}$ & 5 & $11.6 \mathrm{~b}$ & 14 & $3.5 \mathrm{~b}$ & 15 & $6.4 \mathrm{~b}$ & 60 & $16.3^{* *}$ \\
\hline & gro1-2-P40-500 & $\mathrm{IX}_{\mathrm{sd}}$ & Prp1-210 & $-22.7 \mathrm{a}$ & 8 & $14.0 \mathrm{~b}$ & 12 & $4.6 \mathrm{~b}$ & 17 & $6.2 \mathrm{~b}$ & 58 & $16.0^{* *}$ \\
\hline & gro1-2-P40-500 & $\mathrm{XI}_{\mathrm{p}}$ & 683-SSCP1 & $-14.4 \mathrm{a}$ & 10 & $13.1 \mathrm{~b}$ & 10 & $5.8 \mathrm{~b}$ & 32 & $5.3 \mathrm{~b}$ & 39 & $10.1 * *$ \\
\hline & gro 1-2-P40-500 & $\mathrm{XI}_{\mathrm{ld}}$ & GP125-SSCP1 & $-20.2 \mathrm{a}$ & 10 & $18.9 \mathrm{~b}$ & 10 & $4.4 \mathrm{~b}$ & 12 & $6.3 \mathrm{~b}$ & 62 & $18.0^{* *}$ \\
\hline & gro1-2-P40-500 & $\mathrm{XI}_{\mathrm{ld}}$ & NL27-SSCP5 & $-13.9 \mathrm{a}$ & 12 & $19.3 \mathrm{~b}$ & 8 & $5.0 \mathrm{~b}$ & 36 & $6.4 \mathrm{~b}$ & 37 & $13.5^{* *}$ \\
\hline \multirow{3}{*}{$\mathrm{IX}_{\mathrm{sd}}$} & Prp1-210 & $\mathrm{VIII}_{\mathrm{ld}}$ & RC5T3-RsaI-280 & $-17.1 \mathrm{a}$ & 14 & $12.4 \mathrm{~b}$ & 11 & $10.8 \mathrm{~b}$ & 30 & $5.0 \mathrm{~b}$ & 40 & $18.9 * * *$ \\
\hline & Prp1-210 & $\mathrm{XI}_{\mathrm{ld}}$ & NL27-SSCP4b & $1.7 \mathrm{ab}$ & 14 & $-16.2 \mathrm{a}$ & 9 & $5.4 \mathrm{~b}$ & 62 & $23.5 \mathrm{~b}$ & 8 & $15.6 * *$ \\
\hline & Prp1-210 & $\mathrm{XI}_{\mathrm{ld}}$ & NL27-SSCP7 & $-21.0 \mathrm{a}$ & 6 & $0.3 \mathrm{~b}$ & 17 & $13.8 \mathrm{~b}$ & 15 & $5.8 \mathrm{~b}$ & 55 & $12.6^{* *}$ \\
\hline \multirow[t]{3}{*}{$\mathrm{XII}_{\mathrm{ld}}$} & GP34-SSCP2 & $\mathrm{XI}_{\mathrm{p}}$ & 683-SSCP2 & - & 0 & $-20.2 \mathrm{a}$ & 9 & $-5.2 \mathrm{a}$ & 17 & $9.8 \mathrm{~b}$ & 66 & $20.5 \S \S$ \\
\hline & Rx1r1-SSCP1 & $\mathrm{V}_{\mathrm{n}}^{\mathrm{r}}$ & BA121o1t7-SSCP4 & $-22.5 \mathrm{a}$ & 8 & $8.5 \mathrm{~b}$ & 33 & $7.6 \mathrm{ab}$ & 8 & $6.5 \mathrm{~b}$ & 33 & $15.0^{* *}$ \\
\hline & Rx1r1-SSCP2 & $\mathrm{XI}_{\mathrm{ld}}$ & GP125-SSCP1 & $-11.0 \mathrm{a}$ & 11 & $10.9 \mathrm{~b}$ & 41 & $8.9 \mathrm{ab}$ & 8 & $2.8 \mathrm{ab}$ & 23 & $13.2 * *$ \\
\hline
\end{tabular}

${ }^{\mathrm{w}}$ The map segment (MS) tagged by the markers is indicated by chromosome number and suffixes $\mathrm{p}$, ld, and sd for proximal or distal position on the long and short chromosome arm, respectively (Dong et al. 2000).

${ }^{\mathrm{x}}$ Mean MCR and number of individuals (n) that have neither of the two marker alleles (00), only marker allele $2(02)$, only marker allele $1(10)$, or both marker alleles (12). Negative means indicate increased resistance.

${ }^{y}$ Groups of individuals labeled with different lower case letters (a or b) are different $(P \leq 0.05)$ from each other.

$\mathrm{z} * *$ indicates $P \leq 0.01, * * *$ indicates $P \leq 0.001$, and $\S \S$ indicates $P \leq 0.0001$. 


\section{DISCUSSION}

Tagging QTL for late blight resistance in tetraploid potato by targeted genotyping.

Based on linkage maps constructed with RFLP markers for the 24 chromosomes of diploid potato (Gebhardt et al. 1991, 2003; Leonards-Schippers et al. 1994; Tanksley et al. 1992), the genetic length of one complement of the potato genome (12 chromosomes) is in the order of $1,000 \mathrm{cM}$. The genetic length of a map that fully covers the eight complements (96 linkage groups) of a cross between heterozygous tetraploid genotypes should then be approximately $8,000 \mathrm{cM}$. With the liberal assumption that each marker allele can detect effects of QTL located up to $10 \mathrm{cM}$ in distal or proximal direction, a minimum of 400 regularly spaced, polymorphic and singledose markers are required for a genome-wide, fully informative QTL analysis in a tetraploid mapping population. In the two half-sib families derived from crossing the tetraploid cvs. Escort and Nikita with cv. Leyla, 50 nonrandom marker alleles were tested for linkage with QTL, some having simplex and others having duplex allele dosage. The polymorphic markers tagged 11 map segments and only some of the eight homologues per segment that segregated in the F1 families. When testing 50 marker alleles at random, the probability of tagging one QTL with any of the 50 markers is under the favorable circumstances outlined above, 50/400 or 0.12 . The number of QTL segregating in the tetraploid populations is not known. In diploid populations with genome-wide map coverage, no more than 15 QTL for late blight resistance have been detected (Leonards-Schippers et al. 1994; Oberhagemann et al. 1999). With the assumption that 30 QTL segregate in a tetraploid population, three to four of 50 random markers $(0.12 \times 30)$ are expected to detect QTL effects. In the experiment reported, 40 of 50 marker alleles (Table 2), either individually or in pairwise interactions, detected QTL in six and eight map segments of the Escort and Nikita families, respectively. This is far more than expected when using random markers. Targeted genotyping based on 50 marker alleles identified a similar number of QTL as found with a random approach using 337 amplification fragment length polymorphism markers in a different tetraploid cross, which covered approximately $25 \%$ of the genome (Meyer et al. 1998). The criterion for marker selection, which was the position of the markers in map segments previously shown to harbor genes for either quantitative or qualitative resistance, or both, to pathogens in diploid potato, was therefore effective.

However, targeted genotyping identified only part of the genetic variation for resistance to late blight and maturity present in the two families. Marker alleles tagging QTL for maturitycorrected resistance explained up to $38 \%$ of the phenotypic variance in the Nikita family but only up to $15 \%$ in the Escort family. The observed differences can result from genetic differences between the late blight resistant seed parents, the smaller population size of the Escort family when compared to the Nikita family, or both. Escort might be more homozygous than Nikita at the loci tagged by the markers. This is indicated by the smaller number of segregating marker alleles descending from cv. Escort. Alternatively, most resistance QTL segregating in the Escort family are located in map segments that were not tagged by the tested markers.

\section{QTL for plant maturity and resistance to late blight.}

The experiment described in this paper is the first in which QTL for both quantitative resistance to late blight and plant maturity were jointly analyzed in tetraploid breeding materials. After correcting the AUDPC values for maturity, heritable variation for resistance was still detected, indicating that, in the potato genome, factors for resistance to late blight exist that can be separated from the maturity effect. This result is in agreement with a QTL analysis of the same traits in a diploid, unrelated potato population (Visker et al. 2003).

Most map segments having QTL for resistance also harbored QTL for maturity. Why are several, unlinked QTL for resistance to late blight repeatedly linked to QTL for plant maturity? In model one, the genes controlling resistance and maturity are physically linked but structurally and functionally unrelated. The conserved linkage among them results from duplications of the corresponding genomic segments during the evolution of the species. Based on current knowledge on segmental genome duplications in potato (Gebhardt et al. 2003), there is no evidence for duplications that include the reported map segments having both QTL for late blight resistance and maturity. In model two, a large number of unrelated genes control late blight resistance and maturity, which are evenly distributed over the whole potato genome, such that linkage among the QTL is observed by chance alone. This model is not supported by the current knowledge on localization and distribution of QTL for resistance to late blight and maturity on the genetic maps of potato (Collins et al. 1999; Gebhardt and Valkonen 2001; Oberhagemann et al. 1999; Visker et al. 2003). In model three, the effects on resistance to late blight and plant maturity are controlled by pleiotropic alleles of the same gene or by alleles of related, physically linked genes that evolved from common ancestors by local gene duplication and subsequent functional diversification. This model (Collins et al. 1999; Oberhagemann et al. 1999) provides a plausible explanation for the observed linkage between QTL for late blight resistance and maturity and for the variability of QTL effects in the Escort and Nikita families. Marker alleles linked in coupling phase to QTL alleles having

Table 7. Multiple regression analysis with forward selection for MCR (maturity-corrected resistance, mean over years) in populations Escort $\times$ Leyla and Nikita $\times$ Leyla. Phenotypic variance explained by the marker allele $\left(R^{2}\right)$ is shown as percentage

\begin{tabular}{|c|c|c|c|c|}
\hline $\mathbf{M S}^{\mathbf{v}}$ & Marker allele & Origin of allele & Escort $\times$ Leyla & Nikita $\times$ Leyla \\
\hline \multirow[t]{2}{*}{$\mathrm{V}_{\mathrm{p}}$} & $\mathrm{R} 1-1400^{\mathrm{w}}$ & Escort, Nikita & - & $7.2^{* * x}$ \\
\hline & GP179-570y & Escort, Nikita & $7.8^{*}$ & $3.8^{*}$ \\
\hline $\mathrm{VIIII}_{\mathrm{ld}}$ & $57 \mathrm{~T} 3-\mathrm{SSCP}^{\mathrm{z}}$ & Escort, Nikita & - & $4.9^{*}$ \\
\hline $\mathrm{XI}_{\mathrm{p}}$ & $683-\mathrm{SSCP}^{\mathrm{z}}$ & Leyla & - & $7.9^{* *}$ \\
\hline $\mathrm{XI}_{\mathrm{ld}}$ & NL27-SSCP4b ${ }^{z}$ & Leyla & $7.7 *$ & - \\
\hline \multirow[t]{2}{*}{$\mathrm{XII}_{\mathrm{ld}}$} & GP34-SSCP2 ${ }^{\mathrm{z}}$ & Nikita, Leyla & - & $14.3 * * *$ \\
\hline & & & $\Sigma=15.5$ & $\Sigma=38.1$ \\
\hline
\end{tabular}

${ }^{\mathrm{v}}$ The map segment (MS) tagged by the markers is indicated by chromosome number and suffixes $\mathrm{p}$, ld, and sd for proximal or distal position on the long and short chromosome arm, respectively (Dong et al. 2000).

${ }^{\mathrm{w}}$ Marker allele present $=$ plant is more resistant.

x * indicates $P \leq 0.05$, ** indicates $P \leq 0.01$, and *** indicates $P \leq 0.001$.

${ }^{y}$ Presence of the GP179-570 allele increases susceptibility in the Escort family and resistance in the Nikita family.

${ }^{\mathrm{z}}$ Marker allele present $=$ plant is more susceptible. 
a pleiotropic effect on late blight resistance and maturity should show an effect on maturity and AUDPC but not on maturity-corrected resistance. This was indeed observed for few marker alleles, most clearly for P450-SSCP1 in segment $\mathrm{V}_{\mathrm{p}}$ of the Escort family and 683-SSCP1 in segment $\mathrm{XI}_{\mathrm{p}}$ of the Nikita family. Other marker alleles tagged the same map segments but different subsets of the eight homologous chromosomes. This is indicated by the lack of correlation between marker alleles tagging the same map segment and by the different genetic models that fitted marker segregation (Table 2). Some of these marker alleles showed effects on maturity only, others only on resistance, but most markers detected effects on all three traits. This suggests the presence of multiple QTL alleles with variable trait effects in these map segments. Multiple QTL alleles have been shown for a major QTL controlling tuber shape in a cross between diploid, heterozygous potatoes (Van Eck et al. 1994).

\section{Models for the molecular basis \\ of quantitative resistance to late blight.}

$R$ genes and QTL for resistance to various pathogens have been mapped previously to regions $\mathrm{IV}_{\mathrm{sd}}, \mathrm{V}_{\mathrm{p}}, \mathrm{VII}_{\mathrm{p}}, \mathrm{XI}_{\mathrm{ld}}$, and $\mathrm{XII}_{\mathrm{ld}}$ (Fig. 2) (Gebhardt and Valkonen 2001). The same regions of the potato genome contain clustered families of NB-LRR-type genes, some of which have been characterized as functional $R$ genes (Ballvora et al. 2002; Bendahmane et al. 1999, 2000; Hehl et al. 1999; Rickert et al. 2003; Paal et al. 2004; Van der Vossen et al. 2000; Vidal et al. 2002). The markers used for tagging the segments were either derived from NB-LRR-type genes or were closely linked to such genes. In particular, the highly significant marker R1-1400 detects specifically the $R l$ gene for late blight resistance, which is a member of a clustered family of NB-LRR-type genes on potato chromosome V (Ballvora et al. 2002). This suggests that the $R l$ gene family, other NB-LRR-type genes, or both are good candidates for the molecular basis of the resistance QTL in these regions of the potato genome. This model does not rule out the possibility that physically linked but unrelated genes are causal for the observed QTL effects on resistance.

Interlocus interactions were found between marker alleles linked to the NB-LRR-type gene clusters (Tables 5 and 6). Particularly striking were the interactions observed between markers tagging the $R l$ locus for late blight resistance on chromosome $\mathrm{V}$ and seven other loci on different chromosomes, among others the Grol nematode resistance locus on chromosome VII (Paal et al. 2004). Grol occupies a similar position as the Rpil locus for resistance to late blight derived from the wild potato species $S$. pinnatisectum (Kuhl et al. 2001). If NB-LRR-type gene families are causal for resistance QTL, the observed phenotypic interactions among the loci may indicate physical interaction between two or more NB-LRR-type proteins. Specificity and kinetics of pathogen recognition, signal transduction, and defense response could be determined quantitatively by the subunit composition of such recognition complexes. The qualitative, hypersensitive resistance response would be nothing else than an extremely fast reaction triggered by a recognition complex including an $R$ gene (Robertson 1985). Recognition complexes have been proposed to explain the failure of finding in vitro a specific interaction between a single $R$ gene having a LRR domain and the corresponding avirulence gene product (McDowell and Woffenden 2003). No interaction between pairs of NBLRR-type plant resistance genes has been identified so far at the molecular level.

Further candidates for participating in the control of quantitative resistance to late blight are genes functional in the de- fense response (Leonards-Schippers et al. 1994; Trognitz et al. 2002), such as glutathione S-transferase (Hahn and Strittmatter 1994; Martini et al. 1993; Taylor et al. 1990), encoded at the Prpl locus in map segment $\mathrm{IX}_{\mathrm{sd}}$, genes for phenylalanine ammonia-lyase (Hahlbrock and Scheel 1989), mapping to the same region as Prpl (Gebhardt et al. 1991), and osmotin-like genes located in map segments $\mathrm{VIII}_{\mathrm{ld}}$ and $\mathrm{XI}_{\mathrm{p}}$ (Castillo-Ruiz 2002; Liu et al. 1994, Zhu et al. 1995). Markers tagging map segments with PR genes interacted with each other and with map segments having NB-LRR-type genes. Pathogen recognition and defense responses are connected by networks of signal transduction pathways (McDowell and Woffenden 2003). Allelic variation in one part of the network can have indirect, differential effects on the allelic variation affecting other parts, thereby providing a molecular model for the interaction observed between unlinked resistance QTL.

\section{MAS for late blight resistance in tetraploid potato.}

Potato breeding in temperate Europe aims at the selection of cultivars with high levels of resistance to late blight that is not compromised by late maturity. The results of our study allow researchers to design testable MAS strategies towards this goal but also show some limitations. Multiple regression and interaction analysis of QTL for maturity-corrected resistance showed that this trait may be improved by MAS in progeny of cvs. Escort, Nikita, and Leyla. In fact, a first MAS experiment based on three unlinked markers tested in hybrids of Escort $\times$ Leyla resulted in a group of plant individuals that were significantly more resistant to late blight when having the three favorable marker alleles, compared with a control group that had the unfavorable alleles of the same markers. However, the field resistance observed in these plants in a single season could not yet be corrected for the maturity effect (data not shown). Selection will have to be applied mainly against susceptibility alleles rather than in favor of resistance alleles. The only marker alleles consistently linked to increased MCR were CosA-210 and R11400 , which both tag a resistance "hot spot" in map segment $\mathrm{V}_{\mathrm{p}}$. Both marker alleles are associated with resistance to late blight in a large collection of potato varieties (Gebhardt et al. 2004). Presence of most other marker alleles was linked to higher susceptibility. This is particularly evident from the interactions between marker alleles, where the most resistant genotype class (negative MCR means) was the one that lacked both marker alleles (00 genotype class). Due to prevalence of susceptibility alleles, the frequency of genotypes combining more than two favorable alleles is very low in the Escort and Nikita families. Large numbers of progeny are needed to find them, but this can now be approached based on the DNA-based markers reported in this study.

With the exception of marker GP179, the marker alleles chosen for MAS of MCR by multiple regression and forward selection were different for the two half-sib families. Marker GP179 is linked to the Rl locus and to several resistance QTL (Ballvora et al. 2002; Oberhagemann et al. 1999; Rouppe van der Voort et al. 1998, 2000). The marker allele GP179-570, descending from Escort, was, however, linked to increased susceptibility, whereas the same allele when descending from Nikita was linked to increased resistance. This is due either to a crossing over between the resistance QTL and the marker allele present in both parental genotypes or to nonidentity of the 570-base-pair fragment in the two genotypes. The predictive value of this marker is therefore limited to crosses with cvs. Escort and Nikita. Association studies, as performed for CosA-210 and R1-1400, are required for the marker alleles tagging the other map segments, in order to assess their general predictive value for MAS. 


\section{MATERIALS AND METHODS}

\section{Plant material.}

Two tetraploid S. tuberosum F1 families were analyzed, which segregated for quantitative resistance to late blight and did not show a hypersensitive resistance phenotype against the $P$. infestans isolates used for field inoculations. They are referred to as the Escort and Nikita family and originated from crossing cv. Leyla as pollen parent with cvs. Escort and Nikita as seed parents. Leyla is a susceptible cultivar, whereas Escort and Nikita both have good field resistance to late blight. Escort contains the race-specific genes $R 1, R 2, R 3$, and $R 10$. Nikita has at least $R l$, based on presence of an $R l$-specific PCR marker (Table 2). A single tuber per genotype was harvested from pot-grown seedlings in 1998 and was propagated in the field in the subsequent years under the same phytosanitary measures as seed tubers. Individuals maturing very late or having a very poor growth habit were eliminated from the families before the field evaluations for late blight resistance, leaving 84 and 95 genotypes for the Escort and Nikita families, respectively.

\section{Field assessment of late blight and maturity.}

Field experiments were located at Windeby in northern Germany. The Escort and Nikita families were planted in a randomized block design with two replications and four plants per block. Between each block, an infector row with a semisusceptible, medium early cultivar (cvs. Secura and Granola in 2000 , cv. Satina in 2001 and 2002) was planted (distance between the blocks, $80 \mathrm{~cm}$; distance between the rows, $75 \mathrm{~cm}$ ). After tuber planting ( 7 to 9 weeks), every second plant in the infector row was inoculated with a sporangial suspension of a complex isolate of $P$. infestans that was able to overcome the $R 1$ to R11 late blight resistance genes (provided by B. Schöber-Butin, Biologische Bundesanstalt, Braunschweig, Germany). Inoculum was sprayed on the adaxial surface of the leaf, and the leaf was covered overnight with a plastic bag to keep it humid. To maintain continuous infection pressure throughout the experiment, infector rows separating blocks with later maturing genotypes were infected two weeks after the first inoculation. $P$. infestans spread from the inoculated plants into the evaluation plots, simulating a natural infection. From 2 weeks after the first inoculation onwards, nine assessments were made in 3- or 4-day intervals, using a 1 to 9 scale $(1=0 \%$ infection, $9>90 \%)$ (Cruickshank et al. 1982). Average scores per year were calculated from the replications. Late blight resistance was evaluated in years 2000, 2001 (both families), and 2002 (Nikita family only).

The maturity of uninfected plants grown for multiplication was evaluated two to three times within one growing season, using a 1 to 9 scale ( $1=$ very early and $9=$ very late maturity). The plants were compared with standard potato varieties with known maturity type. Maturity evaluations were carried out in years 1999, 2000, 2001 (both families), and 2002 (Nikita family only).

\section{Isolation of total genomic DNA.}

Freeze-dried leaf tissue $(25 \mathrm{mg})$ was ground to fine powder. Genomic DNA was extracted with the DNeasy plant mini kit (Qiagen, Hilden, Germany), according to the supplier's instructions. Peeled, freeze-dried tubers $(0.7 \mathrm{~g})$ were ground to fine powder and were extracted with $20 \mathrm{ml}$ of $100 \mathrm{mM}$ Tris-HCL, pH 9.5, 1.4 M NaCl, 20 mM EDTA, 2\% (wt/vol) CTAB, 1\% (wt/vol) polyethylenglycerol 6000 , and $0.25 \%$ (vol/vol) 2-mercaptoethanol, at $74^{\circ} \mathrm{C}$ for $20 \mathrm{~min}$. The suspension was mixed with 1 volume of chloroform/isoamyl alcohol (24:1 vol/vol) and was centrifuged. The upper aqueous phase was adjusted to $\mathrm{pH}$
7.0 with $1 \mathrm{M} \mathrm{HCl}$ and was treated with $10 \mu \mathrm{g}$ DNAse-free RNAse per ml. The DNA was purified on Qiagen Tip 100 columns (Qiagen) according to the supplier's instructions.

\section{PCR-based marker analysis.}

The Escort and Nikita families were genotyped with 30 PCRbased markers located on all potato linkage groups except linkage group X (Table 1, Fig. 2). Markers R1 (AF447489), Rx1 (AJ011801), BA121o1t7 (Ballvora et al. 2002), gro1-2-P40 (AY196153), NL25 (AJ009719), and NL27 (AJ009720) were derived from NB-LRR-type genes. Markers STM3016, P450, CosA, GP179, GP125, and GP34 are closely linked to NBLRR-type gene families. Markers RC5t7, RC5t3, RC1t3, 573, $57 \mathrm{t} 3$, and 683 tagged osmotin-like PR loci, and markers E22 and 319 tagged a PR-5 homologous locus (Castillo Ruiz 2002). Marker Prp1 originates from a PR glutathione S-transferase gene (Taylor et al. 1990). Primer information is shown in Table 1. PCR was carried out with the following conditions: $50 \mathrm{ng}$ genomic DNA was amplified in a total volume of $25 \mu \mathrm{l}$ of $20 \mathrm{mM}$ Tris- $\mathrm{HCl}, \mathrm{pH} 8.0,50 \mathrm{mM} \mathrm{KCl}, 1.5 \mathrm{mM} \mathrm{MgCl}_{2}, 0.2 \mathrm{mM}$ each of dATP, dCTP, dGTP, and dTTP (Carl Roth \& Co. KG, Karlsruhe, Germany), $0.3 \mu \mathrm{M}$ of each primer, and $0.03 \mathrm{U}$ of Taq DNA polymerase (Invitrogen, Life Technologies, Karlsruhe, Germany) per $\mu \mathrm{l}$. The reaction conditions were as follows: $1 \times$ at $93^{\circ} \mathrm{C}$ for $2 \mathrm{~min}, 40 \times$ denaturation at $93^{\circ} \mathrm{C}$ for $45 \mathrm{~s}$, annealing at $\mathrm{T}_{\mathrm{a}}$ (specified in Table 1) for $45 \mathrm{~s}$, extension at $72^{\circ} \mathrm{C}$ for $1 \mathrm{~min} 30$ s, $1 \times$ at $72^{\circ} \mathrm{C}$ for $10 \mathrm{~min}$. PCR products of different length (SCAR markers) or allele specific PCR products (ASO markers) were separated on standard agarose gels or MetaPhor agarose (microsatellite marker STM3016) and were stained with ethidium bromide. Homomorphic PCR products were digested with 4-cutter restriction enzymes (Table 1), were separated on standard agarose gels, and were stained with ethidium bromide (CAPS markers) or were analyzed for SSCP (Sanguinetti et al. 1994; Slabaugh et al. 1997).

For SSCP analysis, PCR products were digested with the restriction enzymes MseI (New England Biolabs GmbH, Frankfurt, Germany), AluI, or TaqI (Roche Diagnostics $\mathrm{GmbH}$, Mannheim, Germany) (Table 1). Digests $(4 \mu \mathrm{l})$ were added to 8 $\mu \mathrm{l}$ of denaturing solution $(97 \%$ deionized formamid, $0.01 \mathrm{M}$ $\mathrm{NaOH}, 0.05 \%$ xylene cyanol, $0.05 \%$ bromphenol blue), heated to $94^{\circ} \mathrm{C}$ for $4 \mathrm{~min}$ and put immediately on ice. Samples $(6 \mu \mathrm{l})$ were run on $0.25 \times$ MDE gels (BMA BioWhittaker Molecular Applications, Rockland, ME, U.S.A.) in $53.4 \mathrm{mM}$ Tris-borate, $1.2 \mathrm{mM}$ EDTA buffer, pH 8.0 (0.6× Tris-borate-EDTA), and 5\% glycerol. Gel polymerization was initiated with $0.06 \% \mathrm{~N}, \mathrm{~N}, \mathrm{~N}$, $\mathrm{N}$ '-tetramethylethylenediamine and $0.05 \%$ ammoniumperoxodisulfate (Merck, Darmstadt, Germany). Gels were run at $1.5 \mathrm{~W}$ for $17 \mathrm{~h}$ at room temperature. Silver staining was performed as follows: $3 \mathrm{~min}$ in fixation solution (10\% ethanol and $5 \%$ acetic acid), $5 \mathrm{~min}$ in staining solution $(0.2 \%$ (wt/vol) $\mathrm{AgNO}_{3}, 10 \%$ ethanol, and 5\% acetic acid), $2 \mathrm{~min}$ in deionized water, $20 \mathrm{~min}$ in developing solution $(0.75 \mathrm{M} \mathrm{NaOH}, 0.27 \%$ formaldehyde), $5 \mathrm{~min}$ in fixation solution, and rinsed with deionized water.

\section{Mapping of SSCP fragments.}

The segregation of SSCP fragments P450-SSCP1 and P450SSCP2 (Table 1) was scored as presence or absence in the diploid mapping population F1840 (Gebhardt et al. 2003). The fragments were mapped relative to the existing RFLP marker database, using the software package MAPRF (Ritter et al. 1990).

\section{Data analysis.}

Marker alleles (SCAR, ASO, CAPS, or SSCP fragments) were scored as present or absent. Assuming monogenic inheri- 
tance of a single fragment and presence of the fragment being dominant $(A)$ over absence $(a)$, four allelic states are possible in tetraploid potato: homozygous $(A A A A)$ or heterozygous $A A A a$ (triplex/simplex), AAaa (duplex/duplex), or Aaaa (simplex/triplex). Based on the model of tetrasomic inheritance and absence of double reduction (chromatide segregation), the Mendelian ratios expected in a cross between a heterozygous parent having $A$ and a homozygous parent lacking $A$ (aaaa) are 1:0 (presence versus absence) for an $A A A a$ parent, 5:1 for an $A A a a$ parent, and 1:1 for an Aaaa parent. When two heterozygous plants, both having $A$, are crossed, the segregation ratios expected are 1:0 when one or both parents are $A A A A$ or $A A A a, 35: 1$ when both parents are $A A a a, 11: 1$ when one parent is $A A a a$ and the other Aaaa, and 3:1 when both parents are Aaaa. The observed segregation ratio of each marker allele was tested for goodness of fit to one of these models by a $\chi^{2}$ test. The test model was accepted at $P>0.200$.

The relative AUDPC was calculated from the field assessments, according to Jeger and Viljanen-Rollinson (2001). Missing values were estimated with an analysis of variance using the software PLABSTAT version 2P from July 14, 2001 (copyright by F. H. Utz, University of Hohenheim, Stuttgart, Germany). A regression curve of AUDPC against maturity was computed (Fig. 1) using the statistic software SAS, release 8.02 (SAS Institute Inc., Cary, NC, U.S.A.). For each AUDPC measurement, the vertical distance to the regression curve was then calculated, which resulted in negative values for more resistant plants and positive values for more susceptible plants. The phenotypic data were tested for normal distribution, using the software SPSS 10.0 for Windows (SPSS Inc., Chicago, IL, USA). AUDPC values for years 2001 and 2002 and the mean over all years in the Nikita population were normal distributed after $\mathrm{x}^{4}$ transformation. Maturity values were normal distributed only for the mean values over all years but not in the single years.

To test for significant $(P \leq 0.05)$ effects of single marker alleles on maturity, AUDPC, or MCR, a $t$-test was performed in the cases of normal distributed data. Otherwise, a KruskalWallis test was used. Statistical analysis was omitted when one of the two genotype classes (fragment present or absent) had less than 10 individuals, in order to avoid false positive significance due to the large size difference between the classes. Markers having small effects ( $P$ values between 0.01 and 0.05$)$ were included in Tables 3 and 4 only when the effect was repeatedly observed in two or three test years, in a single year and in the average trait value over all test years, or in both mapping populations. Interactions were analyzed by two-way ANOVA between pairs of marker alleles. The interaction test was not further considered when genotype classes consisted of one to three individuals only. The $\mathrm{H}_{0}$ hypothesis of no interaction was rejected at $P \leq 0.01$.

All marker alleles were used in a multiple regression analysis with forward selection at the $P \leq 0.05$ significance level (SAS FORWARD). In each calculation step, the program chooses the independent variable (marker allele) that has the largest significant effect on the dependent variable (phenotypic data) and keeps this variable in the regression model. This marker selection is repeated until no independent variable has a new significant effect $(P \leq 0.05)$ on the dependent variable.

The $\chi^{2}$ test, $t$-test, two-way ANOVA, Kruskal-Wallis test, and the multiple regression were computed with the statistic software SAS, release 8.02 (SAS Institute Inc.) (Dufner 1992).

\section{ACKNOWLEDGMENTS}

This work was supported by grant 0311791 from the German Federal Ministry for Education and Research (BMBF, Bundesministerium für Bildung und Forschung). A. Rickert, R. A. Castillo Ruiz, and J. Paal ac- knowledge the support by BMBF grant 0312290A, BMZ/CIP (German Federal Ministry for Economic Collaboration and Development/Centro International de la Papa) and GIF (German Israeli Foundation) grant G-566269-12.97, respectively. The authors thank for support G. Weber, University of Hohenheim, Stuttgart, Germany, and F. Salamini, director of the Department for Plant Breeding and Yield Physiology, where part of this work was carried out.

\section{LITERATURE CITED}

Ballvora, A., Ercolano, M. R., Weiss, J., Meksem, K.,. Bormann, C. A Oberhagemann, P., Salamini, F., and Gebhardt, C. 2002. The Rl gene for potato resistance to late blight (Phytophthora infestans) belongs to the leucine zipper/NBS/LRR class of plant resistance genes. Plant J. 30:361-371.

Barone, A., Ritter, E., Schachtschabel, U., Debener, T., Salamini, F., and Gebhardt, C. 1990. Localization by restriction fragment length polymorphism mapping in potato of a major dominant gene conferring resistance to the potato cyst nematode Globodera rostochiensis. Mol. Gen. Genet. 224:177-182.

Bendahmane, A., Kanyuka, K., and Baulcombe, D. C. 1997. High-resolution genetical and physical mapping of the $R x$ gene for extreme resistance to potato virus $X$ in tetraploid potato. Theor. Appl. Genet. 95:153162.

Bendahmane, A., Kanyuka, K., and Baulcombe, D. C. 1999. The $R x$ Gene from potato controls separate virus resistance and cell death responses. Plant Cell 11:781-791.

Bendahmane, A., Querci, M., Kanyuka, K., and Baulcombe, D. C. 2000. Agrobacterium transient expression system as a tool for the isolation of disease resistance genes: Application to the $R x 2$ locus in potato. Plant $\mathrm{J}$. 21:73-81.

Bradshaw, J. E., Hackett, C. A., Meyer, R. C., Milbourne, D., McNicol, J. W., Phillips, M. S., and Waugh, R. 1998. Identification of AFLP and SSR markers associated with quantitative resistance to Globodera pallida (Stone) in tetraploid potato (Solanum tuberosum subsp. tuberosum) with a view to marker-assisted selection. Theor. Appl. Genet. 97:202210.

Brigneti, G., Garcia-Mas, J., and Baulcombe, D. C.1997. Molecular mapping of the potato virus $Y$ resistance gene $\mathrm{Ry}_{\text {sto }}$ in potato. Theor. Appl. Genet. 94:198-203.

Brown, C. R., Yang, C. P., Mojtahedi, H., Santo, G. S., and Masuelli, R. 1996. RFLP analysis of resistance to Columbia root-knot nematode derived from Solanum bulbocastanum in a BC2 population. Theor. Appl. Genet. 92:572-576.

Castillo Ruiz, R. A. 2002. A potato large insert library for isolation of candidate loci for late blight resistance and studies on their genome organization. Ph.D. thesis. University of Cologne, Germany.

Collins, A., Milbourne, D., Ramsay, L., Meyer, R., Chatot-Balandras, C., Oberhagemann, P., De Jong, W., Gebhardt, C., Bonnel, E., and Waugh, R. 1999. QTL for field resistance to late blight in potato are strongly correlated with maturity and vigour. Mol. Breed. 5:387-398.

Colon, L. T., Turkensteen, L. J., Prummel, W., Budding, D. J., and Hoogendoorn, J. 1995. Durable resistance to late blight (Phytophthora infestans) in old potato cultivars. Eur. J. Plant Pathol. 101:387-397.

Cruickshank, G., Stewart, H. E., and Wastie, R. L. 1982. An illustrated assessment key for foliage blight of potatoes. Potato Res. 25:213-214

De Jong, W., Forsyth, A., Leister, D., Gebhardt, C., and Baulcombe, D. C. 1997. A potato hypersensitive resistance gene against potato virus $X$ maps to a resistance gene cluster on chromosome 5. Theor. Appl. Genet. 95:246-252.

Dong F., Song, J., Naess, S. K., Helgeson, J. P., Gebhardt, C., and Jiang, J. 2000. Development and applications of a set of chromosome-specific cytogenetic DNA markers in potato. Theor. Appl. Genet. 101:10011007.

Dufner, J., Jensen, U., and Schumacher, E. 1992. Statistik mit SAS. Teubner, Stuttgart, Germany.

El-Kharbotly, A., Leonards-Schippers, C., Huigen, D. J., Jacobsen, E., Pereira, A., Stiekema, W. J., Salamini, F., and Gebhardt, C. 1994. Segregation analysis and RFLP mapping of the $R 1$ and $R 3$ alleles conferring race-specific resistance to Phytophthora infestans in progeny of dihaploid potato parents. Mol. Gen. Genet. 242:749-754.

El-Kharbotly, A., Palomino-Sánchez, C., Salamini, F., Jacobsen, E., and Gebhardt, C. 1996. R6 and $R 7$ alleles of potato conferring race-specific resistance to Phytophthora infestans (Mont.) de Bary identified genetic loci clustering with the R3 locus on chromosome XI. Theor. Appl. Genet. 92:880-884

Ewing, E. E., Šimko, I., Smart, C. D., Bonierbale, M. W., Mizubuti, E. S. G., May, G. D., and Fry, W. E. 2000. Genetic mapping from field tests of qualitative and quantitative resistance to Phytophthora infestans in a 
population derived from Solanum tuberosum and Solanum berthaultii. Mol. Breed. 6:25-36

Gebhardt, C. and Valkonen, J. P. T. 2001. Organization of genes controlling disease resistance in the potato genome. Annu. Rev. Phytopathol. 39:79-102.

Gebhardt, C., Mugniery, D., Ritter, E., Salamini, F., and Bonnel, E. 1993. Identification of RFLP markers closely linked to the $H 1$ gene conferring resistance to Globodera rostochiensis in potato. Theor. Appl Genet. 85:541-544.

Gebhardt C., Ritter, E., Barone, A., Debener, T., Walkemeier, B. Schachtschabel, U., Kaufmann, H., Thompson, R. D., Bonierbale, M. W., Ganal, M. W., Tanksley, S. D., and Salamini, F. 1991. RFLP maps of potato and their alignment with the homoeologous tomato genome. Theor. Appl. Genet. 83:49-57.

Gebhardt, C., Walkemeier, B., Henselewski, H., Barakat, A., Delseny, M., and Stüber, K. 2003. Comparative mapping between potatoes (Solanum tuberosum) and Arabidopsis thaliana reveals structurally conserved domains and ancient duplications in the potato genome. Plant J. 34:529-541.

Gebhardt, C., Ballvora, A., Walkemeier, B., Oberhagemann, P., and Schüler, K. 2004. Genetic potential assessment in germ plasm collections of crop plants by marker-trait association: A case study for potatoes with quantitative variation of resistance to late blight and maturity type. Mol. Breed. 13:93-102.

Ghislain, M., Trognitz, B., Herrera, M. D., Solis, J., Casallo, G., Vasquez, C., Hurtado, O., Castillo, R., Portal, L., and Orrillo, M. 2001. Genetic loci associated with field resistance to late blight in offspring of Solanum phureja and S. tuberosum grown under short-day conditions. Theor. Appl. Genet. 103:433-442.

Hahlbrock, K., and Scheel, D. 1989. Physiology and molecular biology of phenylpropanoid metabolism. Annu. Rev. Plant Physiol. 40:347-369.

Hahn, K., and Strittmatter, G. 1994. Pathogen-defence gene prp1-1 from potato encodes an auxin-responsive glutathione S-transferase. Eur. J. Biochem. 226:619-626.

Hämäläinen, J. H., Watanabe, K. N., Valkonen, J. P. T., Arihara, A., Plaisted, R. L., Pehu, E., Miller, L., and Slack, S. A. 1997. Mapping and marker-assisted selection for a gene for extreme resistance to potato virus $Y$. Theor. Appl. Genet. 94:192-197.

Hämäläinen, J. H., Kekarainen, T., Gebhardt, C., Watanabe, K. N., and P. Valkonen, J. 2000. Recessive and dominant genes interfere with the vascular transport of Potato virus A in diploid potatoes. Mol. PlantMicrobe Interact. 13:402-412.

Hehl, R., Faurie, E., Hesselbach, J., Salamini, F., Whitham, S., Baker, B., and Gebhardt, C. 1999. TMV resistance gene $\mathrm{N}$ homologues are linked to Synchytrium endobioticum resistance in potato. Theor. Appl. Genet.98:379-386.

Jacobs, J. M. E., van Eck, H. J., Horsman, K., Arens, P. F. P., VerkerkBakker, B., Jacobsen, E., Periera, A., and Stiekema, W. J. 1996. Mapping of resistance to the potato cyst nematode Globodera rostochiensis from the wild potato species Solanum vernei. Mol. Breed. 2:51-60.

Jeger, M. J., and Viljanen-Rollinson, S. L. H. 2001. The use of the area under the disease-progress curve (AUDPC) to assess quantitative disease resistance in crop cultivars. Theor. Appl. Genet. 102:32-40.

Kreike, C. M., de Koning, J. R. A., Vinke, J. H., van Ooijen, J. W., and Stiekema, W. J. 1994. Quantitatively-inherited resistance to Globodera pallida is dominated by one major locus in Solanum spegazzinii. Theor. Appl. Genet. 88:764-769.

Kuhl, J. C., Hanneman Jr., R. E., and Havey, M. J. 2001. Characterization and mapping of Rpi1, a late-blight resistance locus from diploid (1EBN) Mexican Solanum pinnatisectum. Mol. Gen. Genet. •••:977-985.

Leonards-Schippers, C., Gieffers, W., Salamini, F., and Gebhardt, C. 1992. The $R 1$ gene conferring race-specific resistance to Phytophthora infestans in potato is located on potato chromosome V. Mol. Gen. Genet. 233:278-283.

Leonards-Schippers, C., Gieffers, W., Schäfer-Pregl, R., Ritter, E., Knapp, S. J., Salamini, F., and Gebhardt, C. 1994. Quantitative resistance to Phytophthora infestans in potato: A case study for QTL mapping in allogamous plant species. Genetics 137:67-77.

Li, X., van Eck, H. J., Rouppe van der Voort, J. N. A. M., Huigen, D.-J., Stam, P., and Jacobsen, E. 1998. Autotetraploids and genetic mapping using common AFLP markers: The $R 2$ allele conferring resistance to Phytophthora infestans mapped on potato chromosome 4. Theor. Appl. Genet.96:1121-1128.

Liu, D., Raghothama, K. G., Hasegawa, P. M., and Bressan, R. A. 1994. Osmotin overexpression in potato delays development of disease symptoms. Proc. Natl. Acad. Sci. U.S.A. 91:1888-1892.

Marczewski, W., Flis, B., Syller, J., Schafer-Pregl, R., and Gebhardt, C. 2001. A major quantitative trait locus for resistance to Potato leafroll virus is located in a resistance hotspot on potato chromosome XI and is tightly linked to N-gene-like markers. Mol. Plant-Microbe Interact. 14:1420-1425.
Martini, N., Egen, M., Rüntz, I., and Strittmatter, G. 1993. Promoter sequences of a potato pathogenesis-related gene mediate transcriptional activation selectively upon fungal infection. Mol. Gen. Genet. 236:179186.

McDowell, J. M., and Woffenden, B. J. 2003. Plant disease resistance genes: Recent insights and potential applications. Trends Biotechnol. 21:178-183.

Meksem, K., Leister, D., Peleman, J., Zabeau, M., Salamini, F., and Gebhardt, C. 1995. A high-resolution map of the vicinity of the $R 1$ locus on chromosome $\mathrm{V}$ of potato based on RFLP and AFLP markers. Mol. Gen. Genet. 249:74-81.

Meyer, R. C., Milbourne, D., Hackett, C. A., Bradshaw, J. E., McNichol, J. W., and Waugh, R. 1998. Linkage analysis in tetraploid potato and association of markers with quantitative resistance to late blight (Phytophthora infestans). Mol. Gen. Genet. 259:150-160.

Milbourne, D., Meyer, R. C., Collins, A. J., Ramsay, L. D., Gebhardt, C., and Waugh, R. 1998. Isolation, characterisation and mapping of simple sequence repeat loci in potato. Mol. Gen. Genet. 259:233-245.

Naess, S. K., Bradeen, J. M., Wielgus, S. M., Haberlach, G. T., McGrath, J. M., and Helgeson, J. P. 2000. Resistance to late blight in Solanum bulbocastanum is mapped to chromosome 8. Theor. Appl. Genet. 101:697-704.

Niewöhner, J., Salamini, F., and Gebhardt, C. 1995. Development of PCR assays diagnostic for RFLP marker alleles closely linked to Grol and H1, conferring resistance to the root cyst nematode Globodera rostochiensis in potato. Mol. Breed. 1:65-78.

Oberhagemann, P., Chatot-Balandras, C., Schäfer-Pregl, R., Wegener, D., Palomino, C., Salamini, F., Bonnel, E., and Gebhardt, C. 1999. A genetic analysis of quantitative resistance to late blight in potato: Towards marker-assisted selection. Mol. Breed. 5:399-415.

Paal, J., Henselewski, H., Muth, J., Meksem, K., Menéndez, C. M., Salamini, F., Ballvora, A., and Gebhardt, C. 2004. Molecular cloning of the potato Grol gene conferring resistance to pathotype Ro1 of the root cyst nematode Globodera rostochiensis based on a candidate gene approach. Plant J. 38:285-297.

Pflieger, S., Lefebvre, V., and Causse, M. 2001. The candidate gene approach in plant genetics: A review. Mol. Breed. 7:275-291.

Pineda, O., Bonierbale, M. W., Plaisted, R. L., Brodie, B. B., and Tanksley, S. D. 1993. Identification of RFLP markers linked to the H1 gene conferring resistance to the potato cyst nematode Globodera rostochiensis. Genome 36:152-156.

Rickert, A. M., Kim, J. H., Meyer, S., Nagel, A., Ballvora, A., Oefner, P. J., and Gebhardt, C. 2003. First-generation SNP/InDel markers tagging loci for pathogen resistance in the potato genome. Plant Biotechnol. J. $1: 399-410$.

Ritter, E., Gebhardt, C., and Salamini, F. 1990. Estimation of recombination frequencies and construction of RFLP linkage maps in plants from crosses between heterozygous parents. Genetics 125:645-654.

Ritter, E., Debener, T., Barone, A., Salamini, F., and Gebhardt, C. 1991. RFLP mapping on potato chromosomes of two genes controlling extreme resistance to Potato virus $X$ (PVX). Mol. Gen. Genet. 227:81-85.

Robertson, D. S. 1985. A possible technique for isolating genomic DNA for quantitative traits in plants. J. Theor. Biol. 117:1-10.

Ross, H. 1986. Potato breeding: Problems and perspectives. Adv. Plant Breed. Supp. 13.

Rouppe van der Voort, J. N. A. M., Wolters, P., Folkertsma, R., Hutten, R., and van Zandvoort, P. 1997. Mapping of the cyst nematode resistance locus Gpa2 in potato using a strategy based on comigrating AFLP markers. Theor. Appl. Genet.95:874-880.

Rouppe van der Voort, J., Lindeman, W., Folkertsma, R., Hutten, R., Overmars, H., van der Vossen, E., Jacobsen, E., and Bakker, J. 1998. A QTL for broad-spectrum resistance to cyst nematode species (Globodera spp.) maps to a resistance gene cluster in potato. Theor. Appl. Genet. 96:654-661.

Rouppe van der Voort, J., Van der Vossen, E., Bakker, E., Overmars, H., van Zandvoort, P., Hutten, R., Klein Lankhorst, R., and Bakker, J. 2000. Two additive QTLs conferring broad-spectrum resistance in potato to Globodera pallida are localized on resistance gene clusters. Theor. Appl. Genet. 101:1122-1130.

Salaman, R. N. 1985. The potato famine: Its causes and consequences. Pages 289-316 in: The History and Social Influence of the Potato. J. G. Hawkes, ed. Cambridge University Press, Cambridge.

Sandbrink, J. M., Colon, L. T., Wolters, P. J. C. C., and Stiekema, W. J. 2000. Two related genotypes of Solanum microdontum carry different segregating alleles for field resistance to Phytophthora infestans. Mol. Breed. 6:215-225.

Sanguinetti, C. J., Dias Neto, E., and Simpson, A. J. G. 1994. Rapid silver staining and recovery of PCR products separated on polyacrylamide gels. Biotechniques 17:914-921.

Simko, I. 2002. Comparative analysis of quantitative trait loci for foliage 
resistance to Phytophthora infestans in tuber-bearing Solanum species. Am. J. Potato Res. 79:125-132.

Slabaugh, M. B., Huestis, G. M., Leonard, J., Holloway, J. L., Rosato, C. Hongtrakul, V., Martini, N., Toepfer, R., Voetz, M., Schell, J., and Knapp, S. J. 1997. Sequence-based genetic markers for genes and gene families: Single-strand conformational polymorphisms for the fatty acid synthesis genes of Cuphea. Theor. Appl. Genet. 94:400-408.

Song, J., Bradeen, J. M., Naess, S. K., Raasch, J. A., Wielgus, S. M., Haberlach, G. T., Liu, J., Kuang, H., Austin-Phillips, S., Buell, C. R., Helgeson, J. P., and Jiang, J. 2003. Gene $R B$ cloned from Solanum bulbocastanum confers broad spectrum resistance to potato late blight. Proc. Natl. Acad. Sci. U.S.A.100:9128-9133.

Tanksley, S. D., Ganal, M. W., Prince, J. P., de Vicente, M. C., Bonierbale, M. W., Broun, P., Fulton, T. M., Giovannoni, J. J., Grandillo, S., and Martin, G. B. 1992. High density molecular linkage maps of the tomato and potato genomes. Genetics 132:1141-1160.

Taylor, J. T., Fritzemeier, H., Häuser, I., Kombrink, E., and Rohwer, F. 1990. Structural analysis and activation by fungal infection of a gene encoding a pathogenesis-related protein in potato. Mol. Plant-Microbe Interact. 3:72-77.

Trognitz, F., Manosalva, P., Gysin, R., Nino-Liu, D., Simon, R., Herrera, M. D., Trognitz, B., Ghislain, M., and Nelson, R. 2002. Plant defense genes associated with quantitative resistance to potato late blight in Solanum phureja $\times$ dihaploid $S$. tuberosum hybrids. Mol. Plant-Microbe Interact. 15:587-597.

Van Eck, H. J., Jacobs, J. M., Stam, P., Ton, J., Stiekema, W. J., and
Jacobsen, E. 1994 Multiple alleles for tuber shape in diploid potato detected by qualitative and quantitative genetic analysis using RFLPs. Genetics 137:303-309.

Van der Vossen, E. A. G., Rouppe van der Voort, J. N. A. M., Kanyuka, K., Bendahmane, A., Sandbrink, H., Baulcombe, D. C., Bakker, J., Stiekema, W. J., and Klein-Lankhorst, R. M. 2000. Homologues of a single resistance-gene cluster in potato confer resistance to distinct pathogens: A virus and a nematode. Plant J. 23:567-576.

Vidal, S., Cabrera, H., Andersson, R. A., Fredriksson, A., and Valkonen, J. P. T. 2002. Potato gene $Y-1$ is an $N$ gene homolog that confers cell death upon infection with Potato virus $Y$. Mol. Plant-Microbe Interact. 15:717-727.

Visker, M. H. P. W., Keizer, L. C. P., van Eck, H. J., Jacobsen, E., Colon, L. T., and Struik, P. C. 2003. Can the QTL for late blight resistance on potato chromosome 5 be attributed to foliage maturity type? Theor. Appl. Genet.106:317-325.

Wastie, R. L. 1991. Breeding for resistance. Adv. Plant Pathol. 7:193-224.

Zhu, B., Chen, T. H. H., and Li, P. H. 1995. Expression of three osmotinlike protein genes in response to osmotic stress and fungal infection in potato. Plant Mol. Biol. 28:17-26.

\section{AUTHOR-RECOMMENDED INTERNET RESOURCE}

Information system for evaluation data on plant genetic resources: www.genres.de/eva 